\title{
Flight mechanics and control of escape manoeuvres in hummingbirds. I. Flight kinematics
}

\author{
Bo Cheng ${ }^{1, *}$, Bret W. Tobalske ${ }^{2}$, Donald R. Powers ${ }^{3}$, Tyson L. Hedrick ${ }^{4}$, Susan M. Wethington ${ }^{5}$, George T. C. Chiu ${ }^{6}$ \\ and Xinyan Deng ${ }^{6}$
}

\begin{abstract}
Hummingbirds are nature's masters of aerobatic manoeuvres. Previous research shows that hummingbirds and insects converged evolutionarily upon similar aerodynamic mechanisms and kinematics in hovering. Herein, we use three-dimensional kinematic data to begin to test for similar convergence of kinematics used for escape flight and to explore the effects of body size upon manoeuvring. We studied four hummingbird species in North America including two large species (magnificent hummingbird, Eugenes fulgens, $7.8 \mathrm{~g}$, and blue-throated hummingbird, Lampornis clemenciae, $8.0 \mathrm{~g}$ ) and two smaller species (broad-billed hummingbird, Cynanthus latirostris, $3.4 \mathrm{~g}$, and black-chinned hummingbirds Archilochus alexandri, $3.1 \mathrm{~g}$ ). Starting from a steady hover, hummingbirds consistently manoeuvred away from perceived threats using a drastic escape response that featured body pitch and roll rotations coupled with a large linear acceleration. Hummingbirds changed their flapping frequency and wing trajectory in all three degrees of freedom on a stroke-by-stroke basis, likely causing rapid and significant alteration of the magnitude and direction of aerodynamic forces. Thus it appears that the flight control of hummingbirds does not obey the 'helicopter model' that is valid for similar escape manoeuvres in fruit flies. Except for broad-billed hummingbirds, the hummingbirds had faster reaction times than those reported for visual feedback control in insects. The two larger hummingbird species performed pitch rotations and global-yaw turns with considerably larger magnitude than the smaller species, but roll rates and cumulative roll angles were similar among the four species.
\end{abstract}

KEY WORDS: Hummingbird, Escape, Flapping, Pitch, Roll

\section{INTRODUCTION}

Hummingbirds and many flying insects are capable of sustained hovering and performing a suite of remarkably fast aerobatic manoeuvres. These species use high-frequency and high-angle-ofattack wing motions during hovering (Dudley, 2000; Ellington, 1984). This is distinct from the flight of conventional humanengineered aircraft with fixed (Anderson, 2005) or rotary wings (Leishman, 2006) or the forward, cruising flight of most bird species

${ }^{1}$ Department of Mechanical and Nuclear Engineering, Pennsylvania State University, University Park, PA 16802, USA. ${ }^{2}$ Field Research Station at Fort Missoula, Division of Biological Sciences, University of Montana, Missoula, MT 59812, USA. ${ }^{3}$ Biology \& Chemistry Department, George Fox University, Newberg, OR 97132, USA. ${ }^{4}$ Department of Biology, University of North Carolina, Chapel Hill, NC 27599, USA. ${ }^{5}$ Hummingbird Monitoring Network, PO Box 115, Patagonia, AZ 85624, USA. ${ }^{6}$ School of Mechanical Engineering, Purdue University, West Lafayette, IN 47907, USA.

*Author for correspondence (buc10@psu.edu)

(D) B.C., 0000-0002-6982-0811
(Tobalske et al., 2003). Previous research has shown that hummingbirds have converged evolutionarily with insects upon aerodynamic mechanisms and wing kinematics used in steady hovering (Warrick et al., 2005, 2009), but insight is lacking into how hummingbirds use their wings to accomplish high-intensity manoeuvres.

The flapping motions used by hummingbirds and insects, with aerodynamic force production during most of wingbeat cycle (Warrick et al., 2009), should facilitate authority of flight control because it allows for rapid and drastic alterations to the magnitude and direction of flight forces to the extent that the animal can alter its wing kinematics (Read et al., 2016). However, closed-loop control of flapping flight during maximal manoeuvres may impose stringent demands on neural-sensing and motor-control systems. Latencies in sensorimotor transduction (Chang and Wang, 2014; Elzinga et al., 2012; Ristroph et al., 2013) and maximal power of flight muscle (Ellington, 1985; Josephson et al., 2000) could significantly limit manoeuvrability. In this respect, hummingbirds may be better than insects at leveraging locomotive advantages offered by a flapping wing using their more elaborate neural and physiological systems. Hummingbird flight muscles have some anaerobic capacity (Chai and Dudley, 1996), which may allow them to generate significantly higher mass-specific power than insects during burst flight (Chai et al., 1997; Marden, 1994), their skeletomuscular system (Hedrick et al., 2011; Welch and Altshuler, 2009) may provide more degrees of freedom of wing motion to facilitate large changes of manoeuvring forces and moments, and their nervous system has higher computational power compared with insects (Iwaniuk and Wylie, 2007), and, therefore, may offer more-complex, high-level flight control through prediction and planning (Flanagan and Wing, 1997; Mischiati et al., 2015).

Therefore, the flight control of hummingbirds, especially during manoeuvres with near-maximal performance, may represent an ideal example for assessing the locomotive advantages offered by flapping flight. Understanding the manoeuvrability of hummingbirds in relation to complex interactions between the physics of flight and neural sensing and motor systems could lead to significant progress in broader animal-locomotion theory, and could also be valuable for biomimetic design of micro air vehicles using flapping wings (Keennon et al., 2012; Ma et al., 2013; Roll et al., 2015).

Considerable research on hummingbird flight has focused on maximal performance during load-lifting (Chai and Dudley, 1995; Chai and Millard, 1997; Altshuler et al., 2010) and during sexually selected displays (Clark, 2009, 2011), but these studies have not included detailed analyses of wing motion that would be useful for improving understanding of underlying flight mechanics and control. Within the repertoire of aerobatic manoeuvres of hummingbirds, we chose to study escape manoeuvring from a 


\begin{tabular}{|c|c|}
\hline AR & aspect ratio \\
\hline$L_{\text {body }}$ & body length \\
\hline$L_{\text {culmen }}$ & culmen length \\
\hline$L_{\text {tail }}$ & tail length \\
\hline$m$ & body mass \\
\hline$n$ & wingbeat frequency \\
\hline$(p, q, r)$ & body roll, pitch and yaw angular velocity \\
\hline$R$ & wing length \\
\hline$\hat{r}$ & dimensionless wing spanwise location \\
\hline$r_{2}(S)$ & dimensionless radius of second moment of wing area \\
\hline $\operatorname{Re}$ & Reynolds number \\
\hline$\hat{t}$ & dimensionless time \\
\hline$t_{0}$ & $\begin{array}{l}\text { start time of the escape manoeuvre (time instant of tail } \\
\text { flaring) }\end{array}$ \\
\hline$t_{\mathrm{f}}$ & end time of the escape manoeuvre \\
\hline$t_{\mathrm{r}}$ & start time of the roll rotation \\
\hline$(u, v, w)$ & body linear velocities along the principal axes \\
\hline$U_{1}$ & stroke-averaged left wing tip velocity \\
\hline$U_{\mathrm{r}}$ & stroke-averaged right wing tip velocity \\
\hline$U_{w}$ & stroke-averaged wing tip velocity at hover \\
\hline$(X, Y, Z)$ & global coordinate frame \\
\hline$\left(x_{\mathrm{b}}, y_{\mathrm{b}}, z_{\mathrm{b}}\right)$ & body coordinate frame \\
\hline$\left(x_{s}, y_{s}, z_{s}\right)$ & horizontal stroke plane coordinate frame \\
\hline$\left(x_{w}, y_{w}, z_{w}\right)$ & wing coordinate frame \\
\hline$\gamma$ & stroke plane angle \\
\hline$\theta$ & wing stroke deviation angle \\
\hline$\phi$ & stroke position angle \\
\hline$\Phi$ & wingbeat amplitude \\
\hline$\psi$ & wing rotation angle \\
\hline$\psi_{0}$ & wing rotation angle at wing base \\
\hline$\psi_{\mathrm{tw}}$ & wing twist rate \\
\hline
\end{tabular}

hovering start because: (1) it is characterized by rapid and largeangle rotations about all three body axes (Clark, 2011) and, therefore, should pose significant challenges in flight control and stabilization, a central problem for both natural and human-made flapping-wing fliers; (2) it is an ecologically relevant behaviour which hummingbirds are able to perform from start to finish even in a confined space, and is therefore amenable to laboratory studies; and (3) only minor stimuli are necessary to repeatedly elicit escape manoeuvres from vigilant hummingbirds and hypothetically the associated flight performance could be near maximal.

With the goal of improving the understanding of mechanics and control (see companion paper, Cheng et al., 2016), herein we report on the free-flight body and wing kinematics of hummingbirds, during escape from a perceived threat in which their manoeuvring flight performance should be close to maximal (Jackson and Dial, 2011). We hypothesize that hummingbirds take advantage of their jointed wing-skeleton to produce larger deviations in wing kinematics than those used by insects during startle or looming avoidance manoeuvres, allowing for multi-axis acrobatic manoeuvres. Additionally, we test the effects of body size on manoeuvring performance. Mass-specific power available for loadlifting appears to be invariant with body mass (Altshuler et al., 2004), and this empirical evidence leads to the prediction that capacity for manoeuvring should scale similarly. In contrast, scaling theory (Kumar and Michael, 2012) suggests that smaller species should manoeuvre more easily than larger species so that larger species may require larger wing kinematic changes to achieve similar levels of performance (this has been discussed in a companion paper, Cheng et al., 2016). We address these questions here using detailed examination of the kinematics of escape manoeuvres of four species of hummingbirds that vary in body mass and wing and tail morphology.

\section{MATERIALS AND METHODS}

\section{Bird capture and housing}

We studied four hummingbird species in North America ( $N=2$ males per species; Table 1) including two large species, magnificent [Eugenes fulgens (Swainson 1827), 7.5 and $8.0 \mathrm{~g}$ ] and blue-throated hummingbirds [Lampornis clemenciae (Lesson 1829), 7.8 and $8.1 \mathrm{~g}$ ], and two smaller species, broad-billed (Cynanthus latirostris Swainson 1827, 3.3 and $3.4 \mathrm{~g}$ ) and black-chinned hummingbirds [Archilochus alexandri (Bourcier \& Mulsant 1846), $3.1 \mathrm{~g}$ ]. Capture and experimentation with magnificent and blue-throated hummingbirds occurred at Southwestern Research Station, American Museum of Natural History, Portal, Arizona, USA (altitude $1450 \mathrm{~m}$ ), and with broad-billed and black-chinned hummingbirds in Patagonia, Arizona, USA (altitude $1236 \mathrm{~m}$ ). Birds were captured using modified Russell traps (Russell and Russell, 2001) with soft mesh, and they were housed individually in $1 \times 1 \times 1 \mathrm{~m}$ flight cages with nutrition available ad libitum in the form of Nektar Plus (NEKTON ${ }^{\circledR}$ Günter Enderle, Pforzheim, BadenWürttemberg, Germany) and a $20 \%$ sucrose solution (mass: volume). Capture and housing were permitted by the US Fish and Wildlife Service and the Arizona Game and Fish Department. Birds were released unharmed after 3-5 days in captivity. Animal procedures were approved by the Institutional Animal Care and Use Committees of the University of Montana and Purdue University.

We obtained morphometrics from the animals using standard techniques (Tobalske et al., 1999) immediately after the experiments. We measured body mass $(m)$ using a digital balance with a resolution of $0.01 \mathrm{~g}$. We photographed the animals ventralside down with one wing spread as in mid-downstroke and also on their back, wings folded and with the head relaxed but the beak gently restrained to be in line with the long axis of the body. The lengths of the beak and tail were included in the body length measurements. Note that flexible body segments and feathers introduce some uncertainty.

\section{Experiment setup and protocol}

We conducted experiments with the hummingbirds inside a transparent flight chamber $(87 \times 77 \times 61 \mathrm{~cm})$ made of acrylic plastic with an open-mesh floor and an elevated perch (Fig. 1A). For feeding during the experiments, we used a modified 3-ml plastic syringe ( $1 \mathrm{~cm}$ in cross-sectional diameter) filled with $20 \%$ sucrose solution. The feeder was suspended approximately $22 \mathrm{~cm}$ from the top and next to one side of the chamber. Birds were introduced to the chamber individually after habituation to captivity (indicated by calm perching in their housing cage). The experiments started after the birds demonstrated voluntary, relaxed feeding in the chamber. During the experiments, we monitored for undue stress or abnormal behaviour; if instances were observed, the birds were returned to their housing.

To elicit near-maximal escape performance, escape from steady hovering to another position in the chamber in minimum time, one investigator startled each feeding bird using a $23 \times 31 \mathrm{~cm}$ black clipboard, held outside the chamber $1 \mathrm{~m}$ from the bird. The investigator held the clipboard adducted, perpendicular to the floor, broad side facing the hummingbird. The investigator used both hands to thrust the clipboard horizontally toward the hovering hummingbird. A single investigator produced all startle stimuli in an effort to minimize variance in presentation among trials. After 
Table 1. Morphometrics of the four hummingbird species studied

\begin{tabular}{|c|c|c|c|c|c|c|c|c|c|c|}
\hline Species & Subjects & $m(g)$ & $L_{\text {body }}(\mathrm{mm})$ & $R(\mathrm{~mm})$ & $\mathrm{AR}$ & $r_{2}(\mathrm{~S})$ & $R / L_{\text {body }}$ & $L_{\text {tail }} / L_{\text {body }}$ & $L_{\text {culmen }} / L_{\text {body }}$ & $\begin{array}{l}R^{3} / \mathrm{m} \\
\left(10^{4} \mathrm{~mm}^{3} \mathrm{~g}^{-1}\right)\end{array}$ \\
\hline \multirow[t]{4}{*}{ Blue-throated (Lampornis clemenciae) } & 1 & 7.82 & 129.93 & 78.90 & 7.78 & 0.50 & 0.61 & 0.31 & 0.18 & 6.28 \\
\hline & 2 & 8.09 & 121.43 & 76.40 & 7.30 & 0.51 & 0.63 & 0.32 & 0.19 & 5.51 \\
\hline & Mean & 7.96 & 125.68 & 77.65 & 7.54 & 0.50 & 0.62 & 0.31 & 0.18 & 5.90 \\
\hline & s.d. & 0.19 & 6.01 & 1.77 & 0.34 & 0.00 & 0.02 & 0.00 & 0.01 & 0.54 \\
\hline \multirow[t]{4}{*}{ Magnificent (Eugenes fulgens) } & 1 & 7.49 & 124.72 & 72.60 & 7.53 & 0.50 & 0.58 & 0.31 & 0.21 & 5.11 \\
\hline & 2 & 7.96 & 122.59 & 76.80 & 7.86 & 0.49 & 0.63 & 0.32 & 0.23 & 5.69 \\
\hline & Mean & 7.73 & 123.66 & 74.70 & 7.70 & 0.49 & 0.60 & 0.32 & 0.22 & 5.40 \\
\hline & s.d. & 0.33 & 1.51 & 2.97 & 0.24 & 0.00 & 0.03 & 0.01 & 0.01 & 0.41 \\
\hline \multirow[t]{4}{*}{ Black-chinned (Archilochus alexandri) } & 1 & 3.09 & 79.95 & 46.30 & 8.15 & 0.48 & 0.58 & 0.29 & 0.23 & 3.21 \\
\hline & 2 & 3.13 & 78.47 & 46.80 & 7.19 & 0.50 & 0.60 & 0.29 & 0.24 & 3.27 \\
\hline & Mean & 3.11 & 79.21 & 46.55 & 7.67 & 0.49 & 0.59 & 0.29 & 0.23 & 3.24 \\
\hline & s.d. & 0.03 & 1.05 & 0.35 & 0.68 & 0.01 & 0.01 & 0.00 & 0.00 & 0.04 \\
\hline \multirow[t]{4}{*}{ Broad-billed (Cynanthus latirostris) } & 1 & 3.37 & 94.12 & 53.70 & 7.63 & 0.49 & 0.57 & 0.29 & 0.22 & 4.60 \\
\hline & 2 & 3.32 & 91.54 & 57.10 & 7.59 & 0.50 & 0.62 & 0.28 & 0.25 & 5.61 \\
\hline & Mean & 3.35 & 92.83 & 55.40 & 7.61 & 0.50 & 0.60 & 0.29 & 0.24 & 5.10 \\
\hline & s.d. & 0.04 & 1.82 & 2.40 & 0.03 & 0.01 & 0.04 & 0.00 & 0.02 & 0.72 \\
\hline
\end{tabular}

See the List of symbols and abbreviations for definitions of variables. Values of $R^{3} / \mathrm{m}$ are shown to illustrate the scaling of wing length relative to body mass; a larger $R^{3} / m$ indicates a relatively longer wing span or smaller body mass.

preliminary experiments, this type of startling stimuli was chosen as it was the most effective in generating repeatable, vigorous escape. Previous startle experiments using streamertail hummingbirds
(Trochilus polytmus) (Clark, 2011) found that the presence of the experimenter significantly increased the vigilance of all the birds, and therefore increased the likelihood of maximal performance in

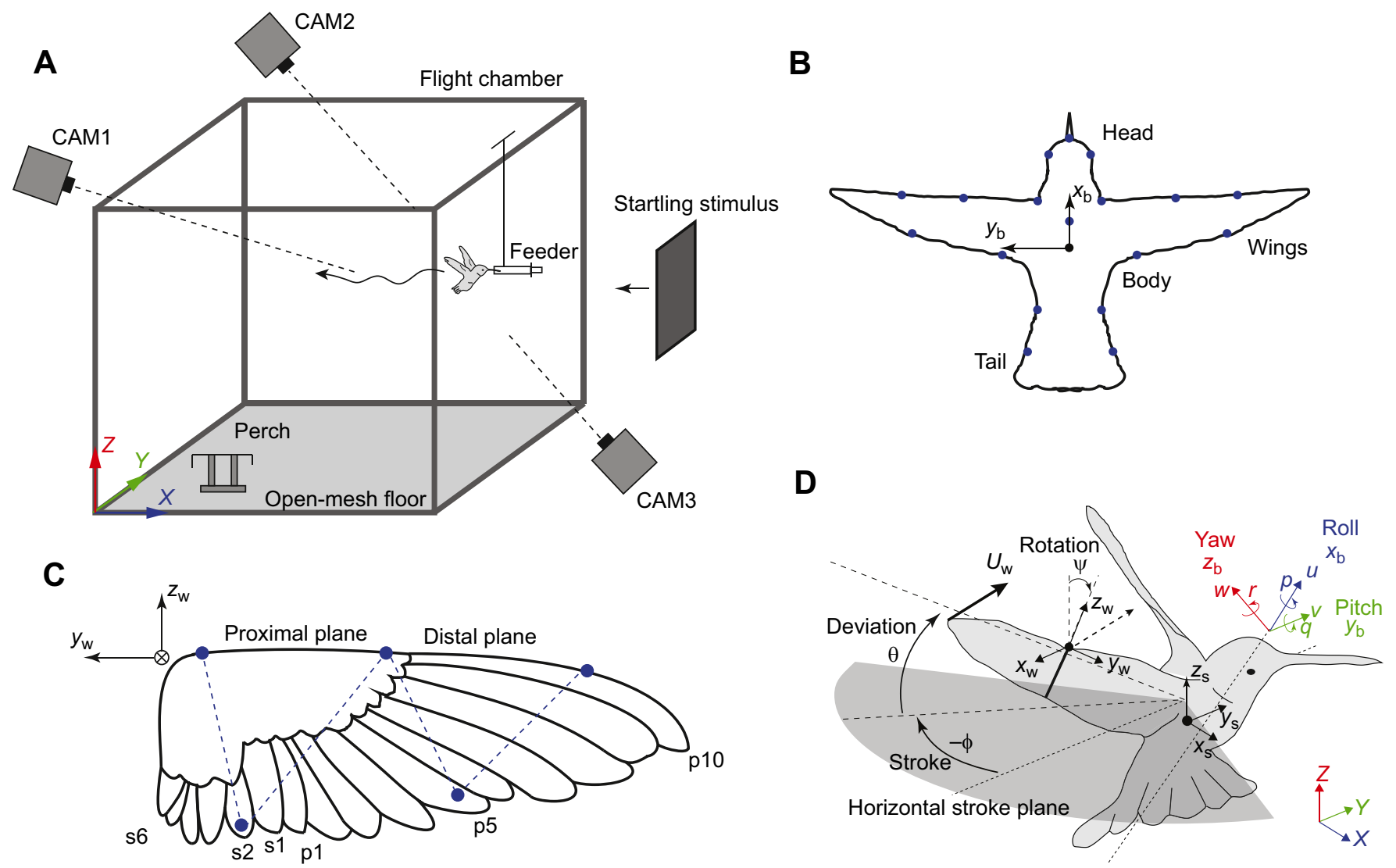

Fig. 1. Experiment setup and definition of kinematics. (A) Experiments were conducted in a flight chamber with an open-mesh floor. Prior to startle, a stationary hovering hummingbird is feeding on a feeder modified from a plastic syringe. The startling stimulus generated by the impulsive movement of a black clipboard elicited the escape manoeuvre. Three synchronized high-speed video cameras were used to record the entire course of the manoeuvre. The global coordinate frame is defined along fore/aft $(X)$, lateral $(Y)$ and vertical $(Z)$ directions. (B) Anatomical landmarks (blue filled dots) used for the extraction of kinematics were located on the head, body, wings and tail of the bird. (C) Details of the anatomical landmarks on the wing show their locations on the primary and secondary feathers and the definitions of proximal and distal planes used for the extraction of wing kinematics. (D) Illustration of body $\left(x_{b}, y_{b}, z_{b}\right)$, horizontal stroke plane $\left(x_{s}, y_{s}, z_{s}\right)$ and wing $\left(x_{w}, y_{w}, z_{w}\right)$ coordinate frames. The wing stroke $(\phi)$, deviation $(\theta)$ and rotation $(\psi)$ angles are defined with respect to the horizontal stroke plane frame. The body linear and angular velocities are specified by $(u, v, w)$ and $(p, q, r)$, respectively. 
escape. No training of the birds for the escape manoeuvres was required. Habituation was not apparent: each bird in a single experiment period was repeatedly startled at least five times, and among these trials no significant order effect was observed. Five trials were recorded on video for each bird, and two of these trials were analyzed quantitatively, giving $N=4$ quantitative samples of kinematics per species.

In separate trials, the startling stimuli were sent to the birds from either frontal or lateral directions. While only the escape manoeuvres from the frontal startles were analyzed quantitatively in this study, qualitative results from the lateral startles were used to explore whether the birds used other distinct manoeuvring patterns.

\section{High-speed videography and kinematics extraction}

We used three synchronized high-speed video cameras, one SA3 and two PCI-1024 (1024×1024 pixel resolution) (Photron Inc., San Diego, CA, USA), sampling at 1000 frames s $^{-1}$ and with a shutter speed of $1 / 6000 \mathrm{~s}$ (for magnificent and blue-throated hummingbirds) and 1/10,000 s (for black-chinned and broadbilled hummingbirds). We illuminated the chamber from outside using four $650-\mathrm{W}$ halogen lights (Lowel Tota-light, Lowel-Light Manufacturing, Brooklyn, NY, USA) and two 50-W LED lights (Fancier LED500, Ningbo Fancier Photographic Equipment Co., Zhenhai, Ningbo, China).

Cameras were calibrated using a direct linear transformation for three-dimensional kinematic reconstruction (Hedrick, 2008). We digitized anatomical landmarks (Fig. 1B) using DLTdv5 (Hedrick, 2008). We marked the birds prior to the experiments using $1.5-\mathrm{mm}$ dots of non-toxic, water-soluble white paint. We placed five markers on each wing (Fig. 1C). In kinematic analyses, proximal and distal wing planes were defined using position vectors based on these points. We placed markers on the lateral tail feathers (Fig. 1B), and defined tail-flaring angle using these points. Head orientation was determined treating the eyes and base of the culmen as points on a plane. Body location was determined as the centroid of markers placed on the feathers over the mid-thoracic spine and each lateral tail junction with the body. The body principal-roll axis $\left(x_{\mathrm{b}}\right)$ was determined using the vector from the averaged location of lateral tail-body junctions to the marker on the spine; pitch axis $\left(y_{\mathrm{b}}\right)$ by averaging two lateral vectors (one connecting lateral tail-body junctions and the other connecting two wing bases); and yaw axis $\left(z_{\mathrm{b}}\right)$ by the crossproduct of the roll and pitch axes (Fig. 1B,D). Horizontal stroke plane frame $\left(x_{\mathrm{s}}, y_{\mathrm{s}}, z_{\mathrm{s}}\right)$ was defined by rotating the body frame by an angle equal to the hovering pitch angle.

Body roll, pitch and yaw Euler angles (using the Fick convention, Murray et al., 1994) were calculated using a rotation matrix from global frame $(X, Y, Z)$ to body frame. Body angular velocities about each principal axis were calculated using the derivative of the rotation matrix (Murray et al., 1994). Time series of body kinematics were low-pass filtered with a cut-off frequency of two times (revealing the within-wingbeat oscillation) or half (no withinwingbeat oscillation) of flapping frequency. Reaction time was calculated as the difference between the start of stimulus (when the clipboard started to move) and tail flaring (e.g. Fig. S1).

\section{Kinematic model of flapping wings}

Coordinate frames rooted at the wings $\left(x_{\mathrm{w}}, y_{\mathrm{w}}, z_{\mathrm{w}}\right)$ were defined according to Fig. 1D. Two sets of wing kinematics, distal and proximal, were obtained using the Euler angles (stroke position $\phi$, stroke deviation $\theta$ and wing rotation $\psi$ ) derived from the rotation matrices from the horizontal stroke plane frame to the distal and proximal wing planes (Fig. 1C,D), respectively. Time series of wing Euler angles were obtained for each trial including the entire period of the manoeuvre and at least three hovering wingbeat cycles prior to manoeuvre. A hovering wing kinematic pattern for each species was obtained by averaging hovering kinematics from four trials. During analysis, we found that the largest changes of wing kinematic angles were coincident with peak angular velocities of the body. Therefore, nominal manoeuvring wing kinematic patterns for generating pitching and rolling moments were obtained for each species by averaging the wingbeat cycles corresponding to peak angular velocities from four trials within a species.

Euler angles of identified wing kinematic patterns were parameterized using a third-order Fourier series prior to further analysis:

$$
\begin{gathered}
\phi(\hat{t})=\phi_{0}+\sum_{i=1}^{3} \phi_{\mathrm{s} i} \sin (2 \pi \hat{i})+\phi_{\mathrm{c} i} \cos (2 \pi i \hat{t}), \\
\theta(\hat{t})=\theta_{0}+\sum_{i=1}^{3} \theta_{\mathrm{s} i} \sin (2 \pi i \hat{t})+\theta_{\mathrm{c} i} \cos (2 \pi i \hat{t}), \\
\psi_{k}(\hat{t})=\psi_{0, k}+\sum_{i=1}^{3} \psi_{\mathrm{s} i, k} \sin (2 \pi i \hat{i})+\psi_{\mathrm{c} i, k} \cos (2 \pi i \hat{t}),
\end{gathered}
$$

where $\hat{t}$ is dimensionless time (varying from 0 to 1 in a wing stroke cycle); $k$ indicates wing rotation angle derived from distal $(k=d)$ or proximal $(k=p)$ planes; and $\psi_{0}, \theta_{\mathrm{s} i}, \psi_{\mathrm{s} i, k}$, etc. are coefficients of the harmonics, which were selected to yield the best least-squares fit to the wing kinematic Euler angles (see Fig. S2 for an example of original data).

Owing to substantial spanwise twist, the distal and proximal wing planes had significantly different wing rotation angles but similar stroke position and deviation angles. To quantify wing twist for blade-element analysis (Cheng et al., 2016), we assumed that all wing chord sections shared the same stroke and derivation angles while having a linearly varying rotation angle from wing base to tip (e.g. a linear twist model; Leishman, 2006; Walker et al., 2009), where local rotation angle of a wing chord section is a linear function of dimensionless spanwise location $\hat{r}(0 \leq \hat{r} \leq 1$, where 0 represents the wing base and 1 represents the wing tip):

$$
\psi(\hat{r}, \hat{t})=\psi_{0}(\hat{t})+\hat{r} \psi_{\mathrm{tw}}(\hat{t}),
$$

where $\psi_{0}$ is wing rotation angle at wing base and $\psi_{\mathrm{tw}}$ is wing twist rate. Both $\psi_{0}$ and $\psi_{\mathrm{tw}}$ are also parameterized by the third-order Fourier series, as they are time-varying functions determined by the rotation angles of proximal and distal wing planes, $\psi_{\mathrm{d}}(t)$ and $\psi_{\mathrm{p}}(t)$ :

$$
\begin{aligned}
\psi_{\mathrm{tw}}(\hat{t}) & =\frac{\psi_{\mathrm{d}}(\hat{t})-\psi_{\mathrm{p}}(\hat{t})}{\hat{r}_{\mathrm{d}}-\hat{r}_{\mathrm{p}}} \\
& =\psi_{0, \mathrm{tw}}+\sum_{i=1}^{3} \psi_{\mathrm{s} i, \mathrm{tw}} \cos (2 \pi i \hat{t})+\psi_{\mathrm{cit \textrm {tw }}} \sin (2 \pi i \hat{t}), \\
\psi_{0}(\hat{t}) & =\frac{-\psi_{\mathrm{d}}(\hat{t}) \hat{r}_{\mathrm{p}}+\psi_{\mathrm{p}}(\hat{t}) \hat{r}_{\mathrm{d}}}{\hat{r}_{\mathrm{d}}-\hat{r}_{\mathrm{p}}} \\
& =\psi_{0,0}+\sum_{i=1}^{3} \psi_{\mathrm{s} i, 0} \cos (2 \pi i \hat{t})+\psi_{\mathrm{c} i, 0} \sin (2 \pi i \hat{t}),
\end{aligned}
$$

where $\hat{r}_{\mathrm{d}}$ and $\hat{r}_{\mathrm{p}}$ are the dimensionless spanwise locations of distal and proximal wing planes, respectively, estimated from the centroids of the corresponding wing planes. We assumed when hummingbirds changed their wing rotation angle during the 
manoeuvres, $\psi_{0}$ remained constant and only $\psi_{\mathrm{tw}}$ changed according to:

$$
\psi_{\mathrm{tw}, \text { manoeuvre }}(\hat{t})=\frac{\psi_{\mathrm{d}, \text { manoeuvre }}(\hat{t})-\psi_{0}(\hat{t})}{\hat{r}_{\mathrm{d}}} .
$$

\section{Statistical analysis of manoeuvring kinematics}

We performed statistical analyses to assess the relationships between changes in wing kinematic patterns and resulting body movements. We computed mean values of wing kinematic variables, including: stroke, deviation, wing rotation, angle of attack, magnitude of wing-tip velocity, stroke-plane angle and flapping frequency. For kinematic variables of the body, we calculated mean linear and angular velocities and accelerations over the interval of $0.15 \mathrm{~s}$ from the start of the manoeuvre, which was time interval of the manoeuvres of shortest duration. Means were computed over the upstrokes and downstrokes separately. For wing kinematic variables, they were based on both bilateral symmetric and asymmetric changes. Hypothetically, bilateral-symmetric changes were used for generating longitudinal body movements (i.e. pitch rotation and fore/aft and vertical translation) and bilateralasymmetric changes were used for generating lateral body movements (i.e. roll and yaw rotation and lateral translation). We computed Pearson's linear correlation coefficients and $P$-values (double-tailed Student's $t$-distribution) between each pair of wing and body kinematic variables using MATLAB (The MathWorks, Natick, MA, USA).

\section{RESULTS}

\section{Morphometrics and scaling}

Our sampling of only four species precluded formal statistical analysis of scaling relationships, so we limit our interpretation to general comparisons with broader samples (Greenewalt, 1962). The four species exhibited almost identical wing planform (Table 1). The two larger species had slightly higher tail-to-body length ratios (Table 1) (consistent with those found in Clark, 2010), suggesting a potentially more important aerodynamic effect of tail in larger species. The black-chinned hummingbirds had relatively wide abdomens and larger body masses than those predicted by isometry, which is evident in their lowest values of $R^{3} / m$ among the species (Table 1).
Wingbeat frequency declined with increasing wing length (Table 2). This was consistent with the broader pattern for insects and hummingbirds, according to the empirical relationship $n R^{1.15}=3540 \mathrm{~mm}^{1.15} \mathrm{~s}^{-1}$ (Greenewalt, 1962); but, again, blackchinned hummingbirds were an exception as they used relatively high wingbeat frequencies for their wing length $\left(n R^{1.15}=\right.$ $4201 \pm 106 \mathrm{~mm}^{1.15} \mathrm{~s}^{-1}$ ), presumably to help support their relatively large body weights. Stroke amplitudes of black-chinned hummingbirds (Table 2) were approximately 10 deg less than those of the other three species. This may be attributed to the physiological constraint that higher wingbeat frequency requires reduced muscle strain (Hedrick et al., 2011). Black-chinned hummingbirds had similar wing length as hawkmoths (Manduca sexta) (Cheng et al., 2011), but they support approximately 2.2 times the body weight and have approximately two times the disc loading (body weight divided by the area swept by wings) using approximately two times the wingbeat frequency. Despite differences in wing lengths and wingbeat frequencies, stroke-averaged wing tip velocities $\left(U_{\mathrm{w}}\right)$ from four species were all $\sim 10 \mathrm{~m} \mathrm{~s}^{-1}$ (Table 2), indicating relatively similar muscle-contraction velocities, but black-chinned hummingbirds had the highest $U_{\mathrm{w}}$ at $\sim 12 \mathrm{~m} \mathrm{~s}^{-1}$.

\section{Body kinematic patterns of escape manoeuvre}

Hummingbirds in each species responded to frontal startling stimulus by steering away using a stereotyped combination of pitch-and-roll manoeuvres (Fig. 2). In few exceptions ( $<5 \%$ of total samples), birds exhibited pitch and backward manoeuvres without changing their heading. We refer to the former as an escape manoeuvre to emphasize that the bird attempted to fly away from the threat by changing its heading and the latter as an avoidance manoeuvre to emphasize that the bird only attempted to back up to avoid the threat.

Both escape and avoidance manoeuvres began with a simultaneous body pitch-up rotation and backward translation. In avoidance manoeuvres, a bird pitched up until its body longitudinal axis was almost aligned with the vertical axis, and then it began to pitch down while decelerating from the backward translation. The pitch-down rotation overshot the hover pitch angle until the body longitudinal axis was almost horizontal, then the bird pitched up again and slowly returned to hover posture. Such a manoeuvring pattern was almost identical to that observed previously in the startle responses of hawkmoths (M. sexta) (Cheng et al., 2011). However,

Table 2. Characteristics of wing kinematics

\begin{tabular}{|c|c|c|c|c|c|c|}
\hline Species & Subjects & $n(\mathrm{~Hz})$ & $\Phi(\mathrm{deg})$ & $U_{w}\left(\mathrm{~m} \mathrm{~s}^{-1}\right)$ & $\operatorname{Re}$ & $n R^{1.15}\left(\mathrm{~mm}^{1.15} \mathrm{~s}^{-1}\right)$ \\
\hline \multirow[t]{4}{*}{ Blue-throated (Lampornis clemenciae) } & 1 & 25.02 & 152.68 & 10.17 & 13,597 & 3801 \\
\hline & 2 & 22.99 & 148.305 & 9.06 & 12,139 & 3366 \\
\hline & Mean & 24.00 & 150.49 & 9.61 & 12,868 & 3583 \\
\hline & s.d. & 1.43 & 3.09 & 0.79 & 1031 & 307 \\
\hline \multirow[t]{4}{*}{ Magnificent (Eugenes fulgens) } & 1 & 22.73 & 156.49 & 8.82 & 11,079 & 3138 \\
\hline & 2 & 26.79 & 154.60 & 11.00 & 13,821 & 3945 \\
\hline & Mean & 24.76 & 155.54 & 9.89 & 12,450 & 3541 \\
\hline & s.d. & 2.87 & 1.34 & 1.54 & 1939 & 571 \\
\hline \multirow[t]{4}{*}{ Black-chinned (Archilochus alexandri) } & 1 & 50.13 & 149.94 & 11.37 & 8796 & 4125 \\
\hline & 2 & 51.32 & 135.69 & 11.77 & 9437 & 4276 \\
\hline & Mean & 50.72 & 142.82 & 11.57 & 9117 & 4201 \\
\hline & s.d. & 0.84 & 10.08 & 0.28 & 454 & 106 \\
\hline \multirow[t]{4}{*}{ Broad-billed (Cynanthus latirostris) } & 1 & 31.25 & 152.52 & 8.86 & 8015 & 3050 \\
\hline & 2 & 31.28 & 151.35 & 9.43 & 9048 & 3276 \\
\hline & Mean & 31.27 & 151.94 & 9.15 & 8532 & 3163 \\
\hline & s.d. & 0.02 & 0.83 & 0.40 & 730 & 160 \\
\hline
\end{tabular}

$U_{\mathrm{w}}$ is stroke-averaged wing tip velocity at hover. $n R^{1.15}$ corresponds to an empirical relation of wingbeat frequency and wing length derived from insects and hummingbirds, $n R^{1.15}=3540\left(\mathrm{~mm}^{1.15} \mathrm{~s}^{-1}\right)$ (Greenewalt, 1962). See List of symbols and abbreviations for definitions of other variables. 
A

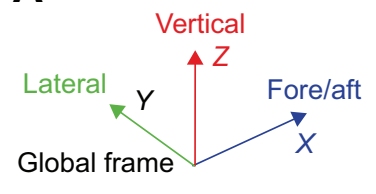

Magnificent hummingbird

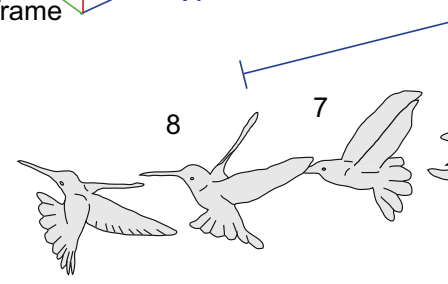

B

E

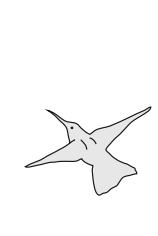

Broad-billed hummingbird

F

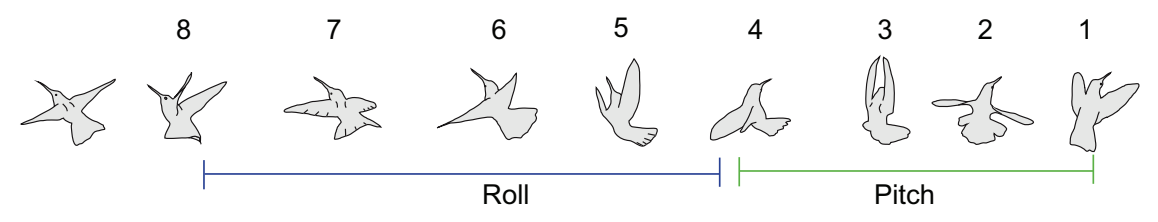

C

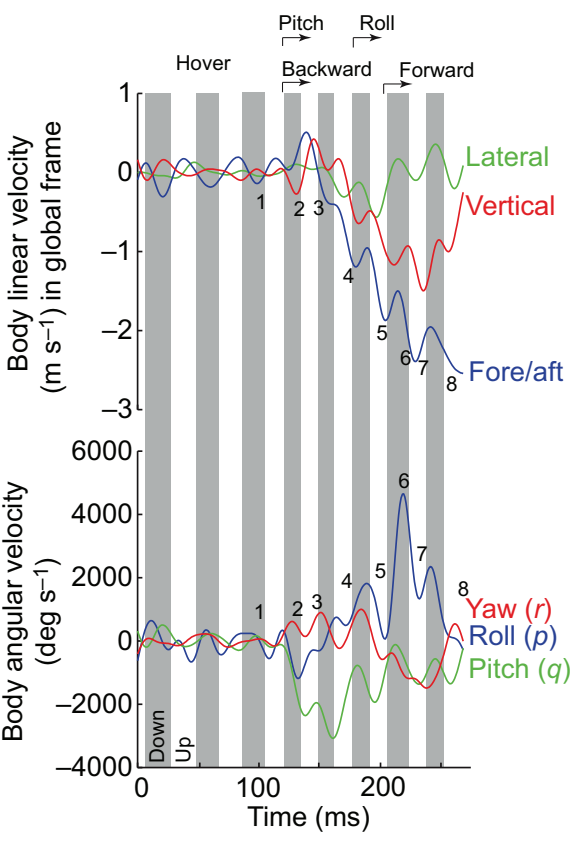

G

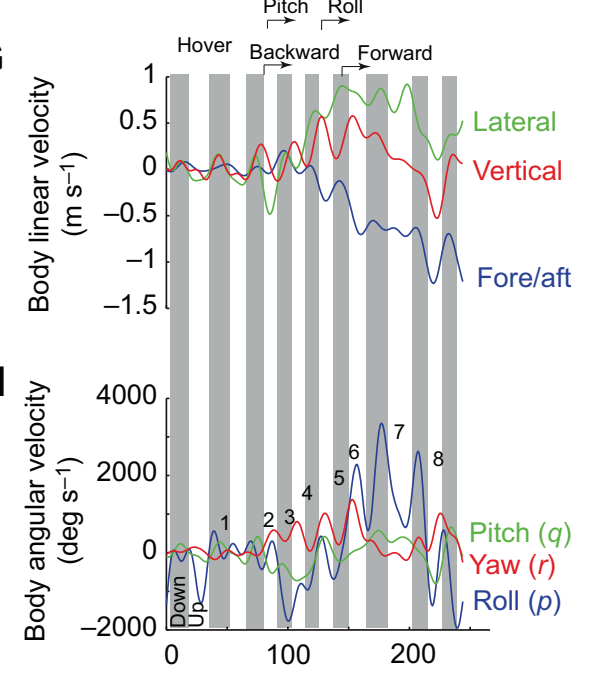

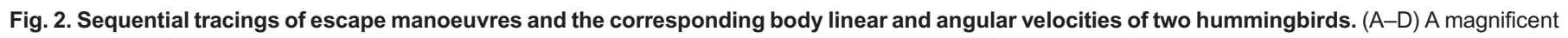
hummingbird. (E-H) A broad-billed hummingbird. (A,E) Tracings from the high-speed videos superimposed to reflect the actual spatial locations of the birds

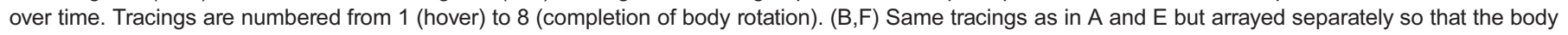

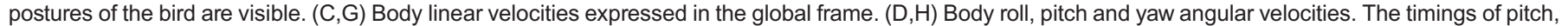
backward acceleration, roll and forward flight are indicated by the arrows on the top of each graph.

avoidance manoeuvres were observed repeatedly in hawkmoths and were the only type of manoeuvre observed in response to a looming startle stimulus. Because of the small number of avoidance manoeuvres observed, they were not further analyzed in the present study.

\section{Pitch-roll escape manoeuvre}

During escape manoeuvres, a hummingbird reoriented its heading through a rapid roll rotation at the second half of the escape manoeuvre, following pitch-up at the first half. This roll not only oriented the bird to the direction of escape, but also led to a smooth transition from backward flight to forward flight without a significant loss of linear momentum.

At the first half of the escape manoeuvre, in addition to the large pitch, minor body roll and yaw were also observed as they oriented the bird to the direction of escape. Concurrent with initial pitch-up, all the birds exhibited a subtle body roll that oriented their dorsum towards the final direction of escape; this was evidenced by a negative roll angular velocity in the beginning of the manoeuvre (blue curves, Fig. 3D,L). There was also a small positive body yaw that oriented the body longitudinal axis towards the direction of escape (red curves, Fig. 3D,L). These two kinematic features increased the linear acceleration towards the direction of escape at the first half of the manoeuvre. This simultaneous roll/yaw body rotation was similar to banked yaw turns (Muijres et al., 2015) or roll/yaw turns (Clark, 2011) described previously for insects and hummingbirds. However, in the escape manoeuvres observed in the present study, they had much smaller magnitude compared with pitch rotations at the first half and the roll rotations at the second half of the manoeuvres. A perfect sequential pitch-roll and a 


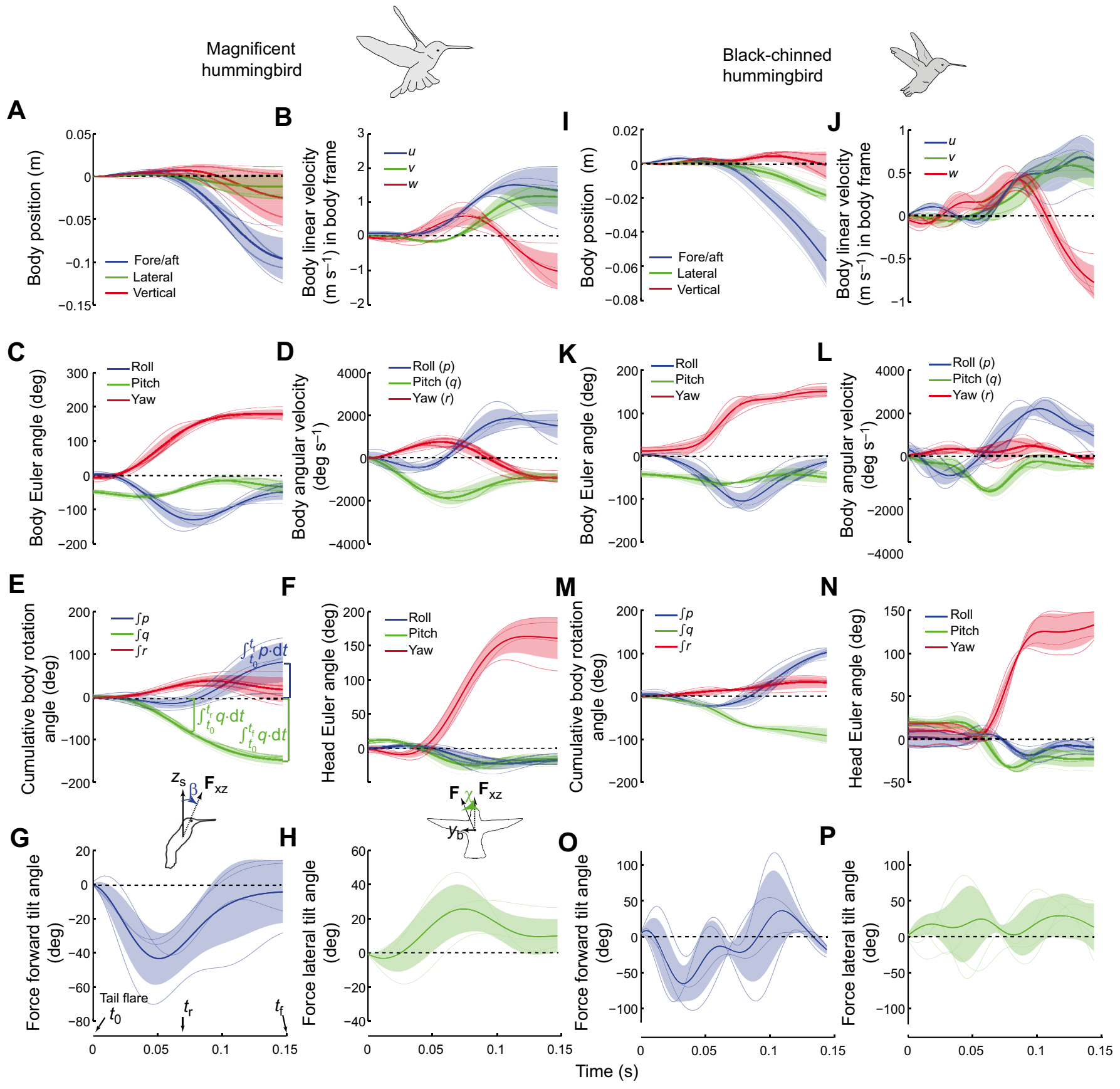

Fig. 3. Body kinematics of four individual escape manoeuvres from two hummingbird species. (A-H) Magnificent hummingbirds. (I-P) Black-chinned hummingbirds. Kinematics include body position $(A, I)$, body linear velocity expressed in the body frame $(B, J)$, body Euler angle $(C, K)$, body angular velocity $(D, L)$, cumulative body rotation angle $(\mathrm{E}, \mathrm{M})$ and head Euler angle relative to the global frame $(\mathrm{F}, \mathrm{N})$. ( $\mathrm{G}, \mathrm{H})$ The longitudinal tilt angle of the aerodynamic force vector, defined as the angle between the normal axis of the horizontal stroke plane $z_{\mathrm{s}}$ and the projection of the force vector on the longitudinal plane $\left(\mathbf{F}_{\mathrm{xz}}\right)$, positive being a forward tilt. $(\mathrm{H}, \mathrm{P})$ The lateral tilt angle of aerodynamic force vector, which is defined as the angle between force vector (F) and its projection on the

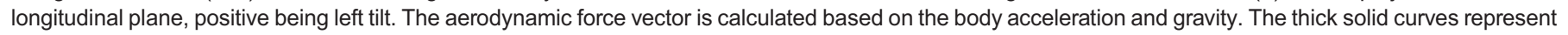
the averaged traces, thin solid curves represent the individual traces, and the shaded areas indicate $\pm 1 \mathrm{~s} . \mathrm{d}$. ( $N=4$ ). The time series of different manoeuvres are aligned at the start of the manoeuvre, which is defined as the instant of tail flaring, and last for $0.15 \mathrm{~s}$, the interval of the manoeuvres of shortest duration.

simultaneous roll/yaw turn are two extremes of the escape manoeuvres observed by Clark (2011); most manoeuvres have body rotations distributed in between. A similar distribution is also observed in escaping fruit flies, as they control the relative amount of roll and pitch to change the escape direction when approached with danger (Muijres et al., 2014). In the present study, we found that the pitch-roll manoeuvres were more dominant than simultaneous roll/yaw when the danger was from the front. The birds performed only a small 'banked turn' while simultaneously pitching up in the first half of the manoeuvre and then rolled back to normal forward flight/hover postures in the second half of the manoeuvre. Because of the dominance of pitch and roll rotation, we use the term 'pitch-roll'.

Stroke-averaged body kinematics showed that global yaw turning angle was strongly correlated with cumulative body pitch angle prior to the start of roll rotation $\left(t_{t_{\mathrm{r}}}^{t_{\mathrm{r}}} q \cdot \mathrm{d} t\right.$, where $q$ is 
pitch rate, and $t_{0}$ and $t_{\mathrm{r}}$ are the starting times of pitch and roll, respectively; Fig. 4A) as well as with the total cumulative body pitch angle over the entire period of the manoeuvre ${ }_{t_{0}}^{t_{\mathrm{f}}} q \cdot \mathrm{d} t$, where $t_{\mathrm{f}}$ is the final time of manoeuvre, defined as when the roll angle was approximately zero at the end of the manoeuvres; Fig. 4B). However, the correlation between the yaw turning angle and the total cumulative roll angle $\left({ }_{t_{0}}^{t_{\mathrm{p}}} p \cdot \mathrm{d} t\right)$, primarily at the second half of the manoeuvre, was relatively weak compared with the former variables (Fig. 4C). Representations of $t_{0}, t_{\mathrm{r}}$ and $t_{\mathrm{f}}$ are shown in Fig. 3E. Because the cumulative body pitch angle prior to the start of the roll rotation reflected the timing of the roll rotation relative to the pitch rotation (or the pitch angle when the bird started to roll), the birds were able to perform global yaw turns with varying magnitude by simply controlling the timing of roll rotation. For example (Fig. 2), compare a 180 deg yaw turn performed by a magnificent hummingbird (larger species) with a 140 deg yaw turn performed by a broad-billed hummingbird (smaller species). Pitch-roll manoeuvres were also used by all of the species in response to lateral-startling stimuli, but in these cases, the manoeuvres had earlier timings of roll rotation compared with those in response to frontal-startling stimuli, thus leading to smaller global yaw turns close to $90 \mathrm{deg}$.

Similar pitch-roll manoeuvres are also observed in hovering male horse flies (Hybomitra hinei wrighti) while they rapidly change direction to chase passing females or any small fast-moving objects (Wilkerson and Butler, 1984), and these manoeuvres were previously compared with the 'Immelmann turn' commonly used in the aerobatic manoeuvres of aircraft to rapidly reposition with 180 deg heading change. Compared with banked yaw turns (Clark, 2011; Muijres et al., 2015), which may create similar global yaw turning angle and end forward velocity, a pitch-roll turn allows a bird to almost instantly burst into backward flight to evade the startle stimulus (or creating larger backward acceleration at the start of the manoeuvre; also see Clark, 2011) and then smoothly transition to forward flight by rolling its body. It also ensures a nearly continuous acceleration towards the direction of escape, but it should require accurate control or preplanning of the timing of roll rotation (Cheng et al., 2016). This interpretation assumes near-maximal flight performance, which is reasonable because the hummingbirds remained vigilant during the experiments. Nonetheless, given the vagaries of motivation possible for wild animals subjected to repeated tests in captivity, caution is warranted in interpreting our conclusion of optimality.

\section{Interspecific differences of body kinematics}

Although four hummingbird species showed stereotyped pitch-roll manoeuvres in response to the startling stimuli, two larger species performed pitch rotations with considerably larger magnitude than smaller species and attained larger global-yaw turns (Fig. 4). Comparing body kinematics of magnificent and black-chinned hummingbirds (Fig. 3), cumulative body pitch angle was greater (Fig. 3E,M) and timing of roll rotation was noticeably later in magnificent hummingbirds. However, peak roll rates (Fig. 3D,L) and cumulative roll angles (Fig. 3E,M) were similar interspecifically. As a result, magnificent hummingbirds performed more drastic escape manoeuvres with larger globalyaw turn (Fig. 3C,K) and higher linear exiting velocities towards the end of manoeuvres (Fig. 3B,J).

\section{Escape reaction time}

Time lag between startling stimulus and tail flaring, which preceded any noticeable changes of wing motion, were less in larger species compared with the smaller species. Reaction times were $21 \pm 1.4 \mathrm{~ms}$ for magnificent hummingbirds, $22.5 \pm 8.3 \mathrm{~ms}$ for blue-throated hummingbirds, $28.5 \pm 8.1 \mathrm{~ms}$ for black-chinned hummingbirds and $53.5 \pm 10.7 \mathrm{~ms}$ for broad-billed hummingbirds. Except for broadbilled hummingbirds, all the birds had reaction times much lower than those reported for the visual feedback of insects. For example, hawkmoths have $>50 \mathrm{~ms}$ delays in responding to moving flowers (Sprayberry, 2009), and fruit flies have $\sim 100 \mathrm{~ms}$ and $\sim 61 \mathrm{~ms}$ delays in responding to gust perturbation and looming-startling stimuli, respectively (Fuller et al., 2014; Muijres et al., 2014). These data reveal that hummingbirds are capable of faster visual processing than insects. The estimated time delays here were comparable to those reported for mechanosensory feedbacks of fruit flies: $\sim 20 \mathrm{~ms}$ in wind sensing (Fuller et al., 2014), 10-25 ms in responding to yaw perturbation (Ristroph et al., 2010) and $10-15 \mathrm{~ms}$ in responding to pitch perturbation (Ristroph et al., 2013).

\section{Wing kinematics of hovering}

Kinematic patterns of the distal wing of hovering hummingbirds, normalized by wingbeat frequency, show virtually no interspecific
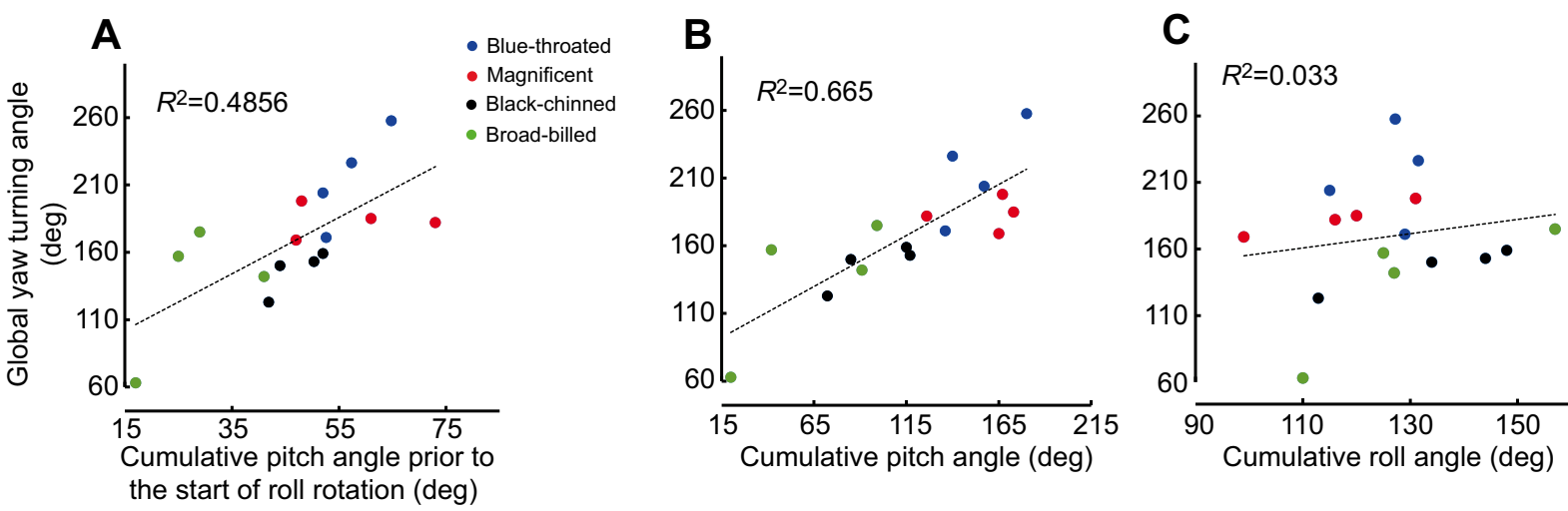

Fig. 4. The relationships between global yaw turning angle and three types of cumulative body rotation angles. (A) Cumulative body pitch angle prior to the start of roll rotation $\left(\int_{t_{0}}^{t_{r}} q \cdot \mathrm{d} t\right)$, which reflects the instantaneous pitch angle at the start of roll rotation or the timing of roll rotation relative the pitch rotation; $(B)$ total cumulative body pitch angle of the entire manoeuvre $\left(\int_{t_{0}}^{t_{f}} q \cdot \mathrm{d} t\right)$, which reflects the magnitude of the pitch rotation; and $(C)$ total cumulative body roll angle $\left(\int_{t_{0}}^{t_{f}} p \cdot \mathrm{d} t\right)$, which reflects the magnitude of the roll rotation. The cumulative body angles are different from the body Euler angles as they were integrated directly from the body angular velocities. Results of the four species are shown in different colours. Linear regressions (dotted lines) show that global yaw heading changes have strong correlations with the timing of roll rotation relative to the pitch rotation $(A)$ and the magnitude of pitch rotation (B), but not with the magnitude of the roll rotation $(\mathrm{C})$ 
differences in time series (Fig. 5A-C) or wing-tip trajectories (Fig. 6). The shallow U-shaped tip trajectories were similar to those measured from insects such as fruit flies (Muijres et al., 2014), drone flies (Liu and Sun, 2008) and hawkmoths (Cheng et al., 2011).

Comparing the kinematics derived from the distal wing planes with those derived from the proximal planes (Fig. 5D-G) revealed substantial differences in the wing rotation angles (Fig. 5E). The distal and proximal wing planes had similar wing stroke and deviation angles, with the proximal section slightly leading the distal section (Fig. 5D); however, there were large discrepancies of wing rotation angle, especially during the upstroke. Thus the wings underwent large spanwise twist but only minor spanwise bending when flapping. Previous studies of hovering hummingbirds (Song et al., 2014; Warrick et al., 2005) have revealed that wing deformations included both chordwise camber and spanwise twist. The time course of the twist rate is presented in Fig. 5H, which shows that the spanwise twist was much more substantial in the upstroke than in the downstroke. Although the magnitudes of wing pronation (in downstroke) and supination (in upstroke) were similar at the distal section (black curve, Fig. 5E), at the proximal section, because of the wing twist, there was much smaller supination than pronation (green curve, Fig. 5E,G). This pattern of wing twist is similar to the 'washout' design used in the blades of propellers and helicopters, which is implemented to reduce the induced-power loss in the wake and therefore increase the aerodynamic efficiency (Leishman, 2006).

A

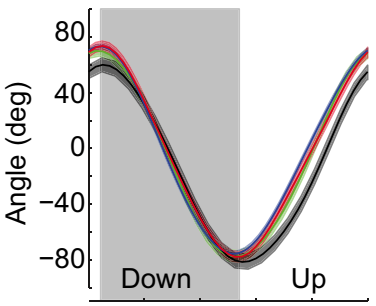

D

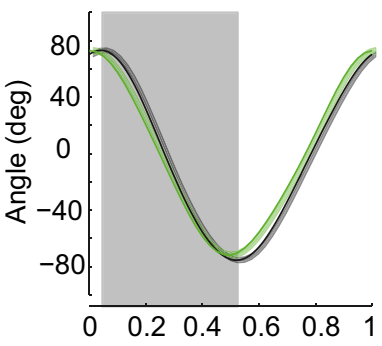

B

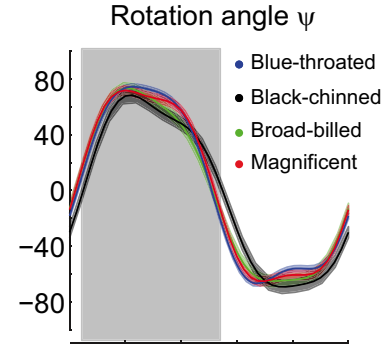

E

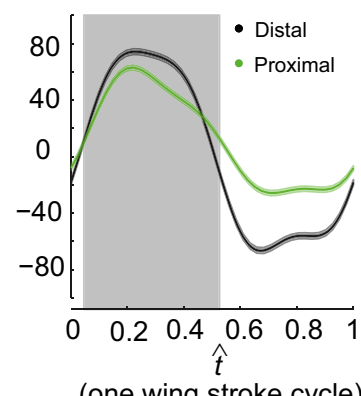

\section{Wing kinematics of escape manoeuvre}

Wing kinematics derived from the distal wing plane showed greater changes from the hovering kinematics than those derived from the proximal plane and, therefore, were better indicators of active changes of wing kinematics for controlling escape manoeuvres. These patterns are novel information for hummingbirds; in comparison with insects, whose wing kinematics during various types manoeuvres are available from previous work (Beatus et al., 2015; Cheng et al., 2011; Fry et al., 2003; Muijres et al., 2014; Ristroph et al., 2010; Taylor, 2001). It has been observed that hummingbirds are able to rapidly generate substantial changes of their wing motion for manoeuvring. The manoeuvring wing kinematics show high temporal and spatial variability within each hummingbird. The spatial variability is shown by the substantial changes of wing motion in all three degrees of freedom, leading to pronounced bilateral symmetric and asymmetric control of wing motion. The temporal variability is shown by the rapid transition between different wing kinematic patterns in different phases of the manoeuvre, which signifies the hummingbirds' ability to quickly change flight control force. As will be described further, below, compared with insects of smaller size, such as fruit flies, such an ability of controlling the motion is required for hummingbirds to generate comparable body angular rates. Arguably, this ability can be made possible for hummingbirds by their powerful flight muscles for both up and down strokes (Tobalske et al., 2010), larger degrees of freedom provided by the proximal forelimb skeletomuscular system (Hedrick et al., 2011; Welch and Altshuler, 2009), and the complex avian nervous system (Scanes, 2014). A
C

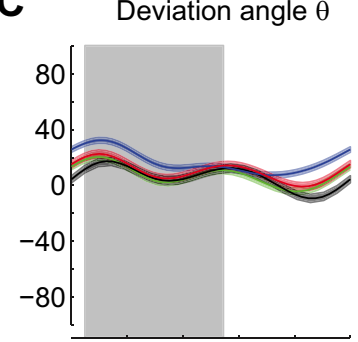

$\mathbf{F}$

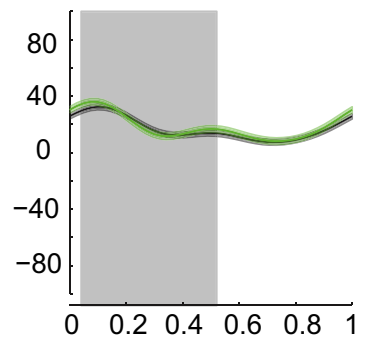

Fig. 5. Wing kinematics in hovering hummingbirds. $(A-C)$ Wing kinematic angles derived from the distal wing plane for the four species: $(A)$ stroke, $(B)$ rotation and (C) deviation. (D-F) Comparison of the wing kinematic angles derived from the distal and proximal wing planes (from blue-throated hummingbird). The shaded areas enclosing the curves indicate \pm 1 s.d. $(N=4)$. (G) Schematic representation of the wing kinematics derived from the proximal and distal wing planes. The line denotes the wing chord, with a circle marking the leading edge. $(\mathrm{H})$ Root rotation angle $\psi_{0}$ (dotted line) and twist rate $\psi_{\mathrm{tw}}$ (solid line) as defined in Eqn 4.
G

Proximal

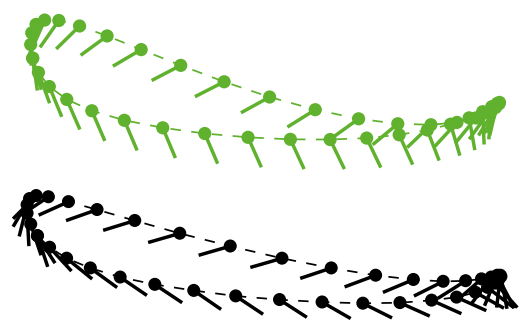

H

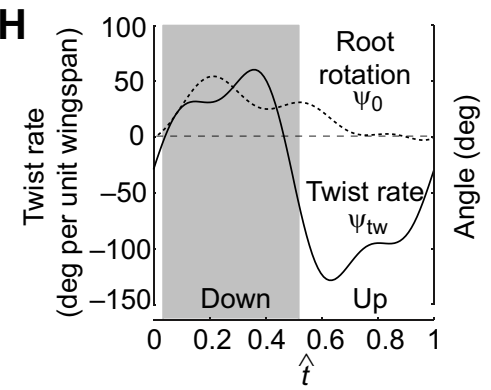



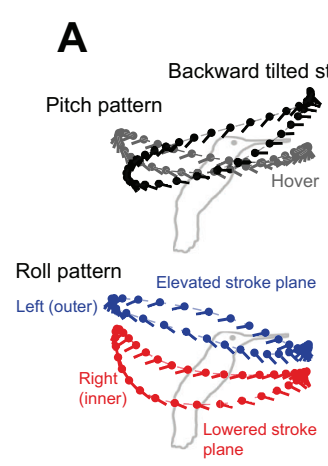

C

Pitch pattern

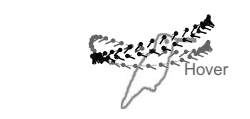

Roll pattern
Left
Magnificent hummingbird

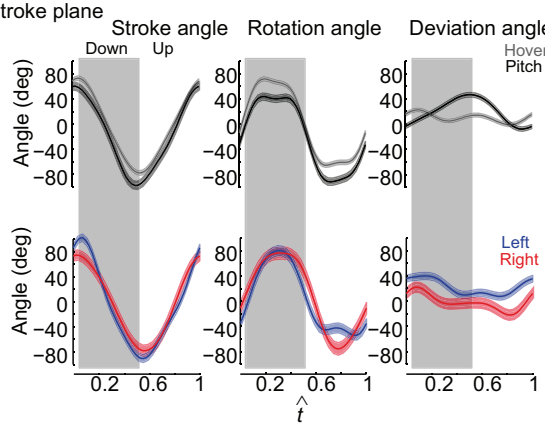

Black-chinned hummingbird

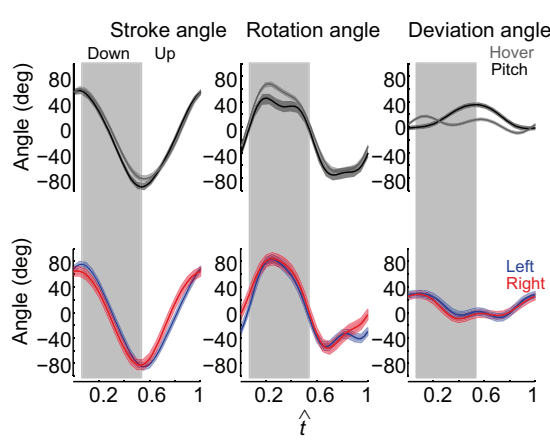

B

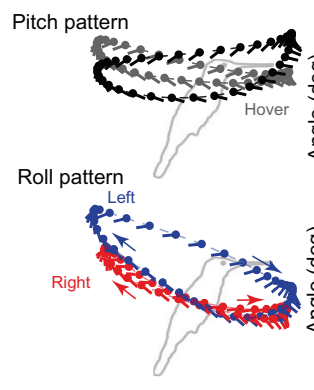

D

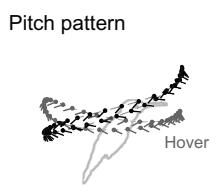

Roll pattern

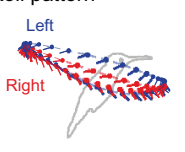

Blue-throated hummingbird

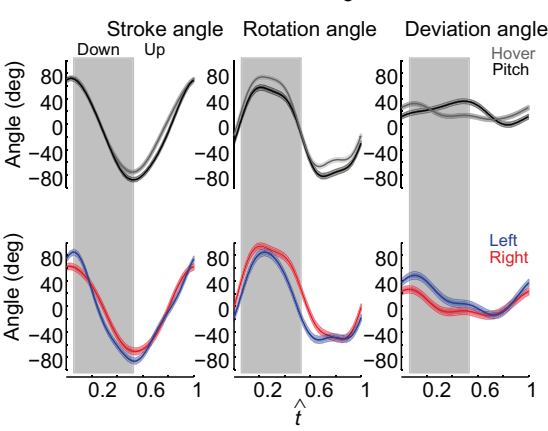

road-billed hummingbird

Stroke angle Rotation angle Deviation angle
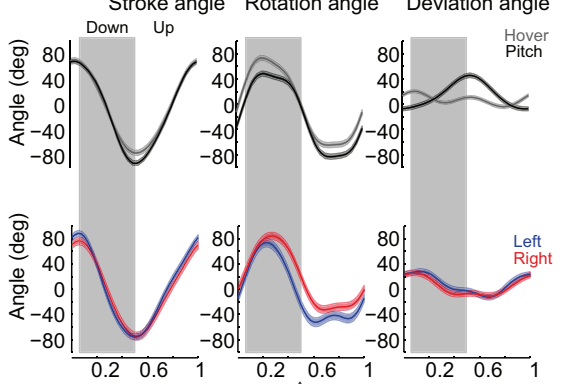

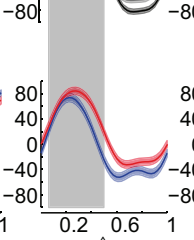

Fig. 6. Wing kinematics in manoeuvring hummingbirds. The kinematics for the four species are shown in four separate panels: (A) magnificent hummingbird, (B) blue-throated hummingbird, (C) black-chinned hummingbird and (D) broad-billed hummingbird. In each panel, schematic representation of the wing kinematics during pitch and roll rotation are shown on the left and the corresponding time series of stroke, rotation and deviation angles are shown on the right.

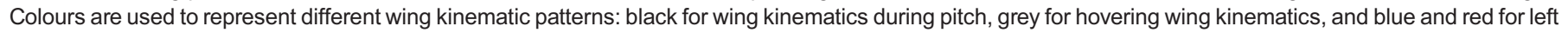
(inner) and right (outer) wing kinematics during roll. The shaded areas enclosing the curves indicate $\pm 1 \mathrm{~s} . \mathrm{d}$. $(N=4)$.

complete summary of body and wing kinematic time series from an example escape manoeuvre can be found in Fig. S1.

Correlating mean wing kinematics with body movement, we observed significant trends intraspecifically, but only a few correlations were significant interspecifically (Tables S1-S4). Salient wing kinematic changes in all four species were apparent in wing-tip trajectories (Fig. 6) and were consistent with the interspecifically-significant correlations. Wing kinematics were correlated primarily with the body angular velocities instead of accelerations, consistent with previous studies on avoidance manoeuvres in hawkmoths (Cheng et al., 2011). This was at least partly due to the use of wing stroke-averaged kinematic variables, as any within-wingbeat acceleration and deceleration will cancel. Bilateral-symmetric changes were correlated with pitch and bilateral-asymmetric changes were correlated with roll and yaw (Table S1).

The size of the flight chamber may have affected the performance of escape manoeuvres compared with previous studies in larger arenas (Clark, 2011; Segre et al., 2015). The birds of different sizes were able to complete a sequence of stereotyped body rotations and achieve comparable forward velocity at the end of the manoeuvres; however, they started to decelerate within two wingbeats after completing their body rotations. In previous studies, the birds continued flying forward (Clark, 2011; Segre et al., 2015). Although the size of the chamber did not affect the completion of the escape manoeuvres, it may have increased the propensity to use of a particular type of manoeuvre, namely pitch-roll manoeuvres compared with roll/yaw manoeuvres (Clark, 2011) and arcing turns (Segre et al., 2015), both of which require larger space for both fore/ aft and lateral translation.

\section{Wing kinematics for pitch rotation}

In the beginning of an escape manoeuvre, hummingbirds rapidly pitched up and accelerated backwards; this was achieved by employing bilateral-symmetric wing kinematic changes including: a backward tilt of stroke plane, a backward shift in mean spanwise rotation angle and a slight forward shift of mean stroke angle (Fig. 6). These kinematic changes were also accompanied by an increase of wingbeat frequency and speed. There was a significant correlation between the above-wing kinematic variables and the stroke-averaged pitch rate (Fig. 7). The manoeuvring forces and moments resulted from these kinematic changes can be inferred without detailed calculation of aerodynamic forces. Specifically, the backward tilt of stroke plane oriented the mean lift force backward, creating lift-based pitch-up moment and backward force; the backward shift of mean spanwise rotation angle (reduced pronation and enhanced supination) led to a higher angle of attack and drag during the downstroke, and a lower angle of attack and drag during upstroke, thus creating drag-based pitch-up moment and backward force; and the forward shift of mean stroke plane angle led to an imbalance of lift with respect to the centre of mass, thus creating a lift-based pitch moment without creating much backward force. Collectively, it can be seen that the hummingbirds exploited all the major kinematic changes possible for generating pitch moment and backward forces.

During escape manoeuvres, hummingbirds significantly increased their wingbeat frequencies (e.g. indicated by the reduced width of shaded areas in Fig. 2C,D,G,H). Larger species increased their frequency by approximately $50 \%$ relative to hovering. Such drastic increases in wingbeat frequency were not observed in previous maximal load-lifting experiments in the 

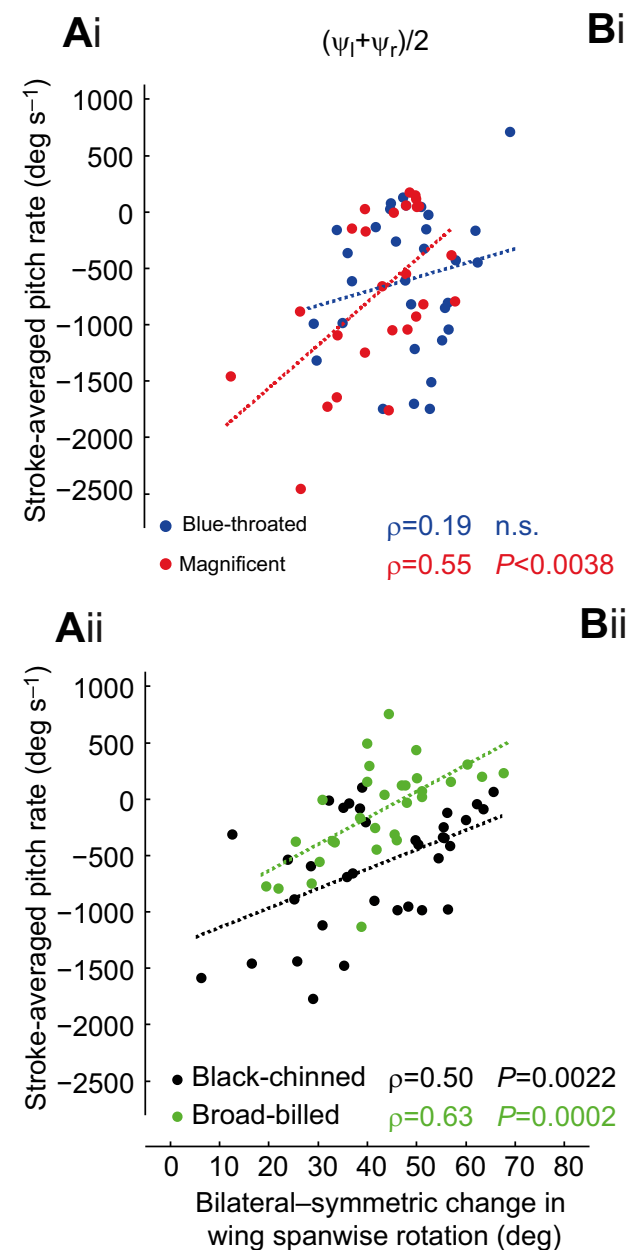

$\mathrm{Bi}$

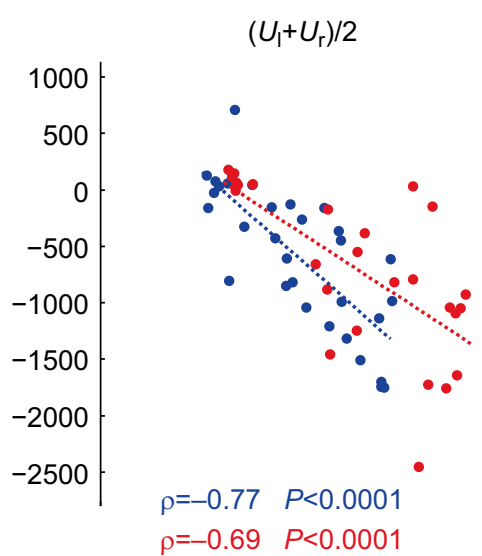

iii

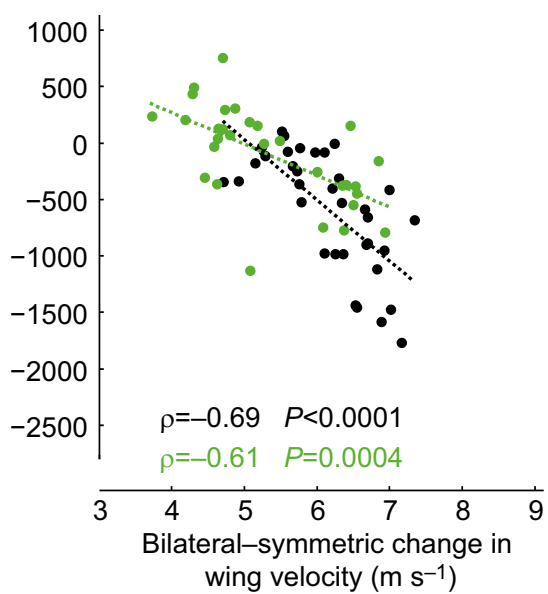

$\mathbf{C i}$

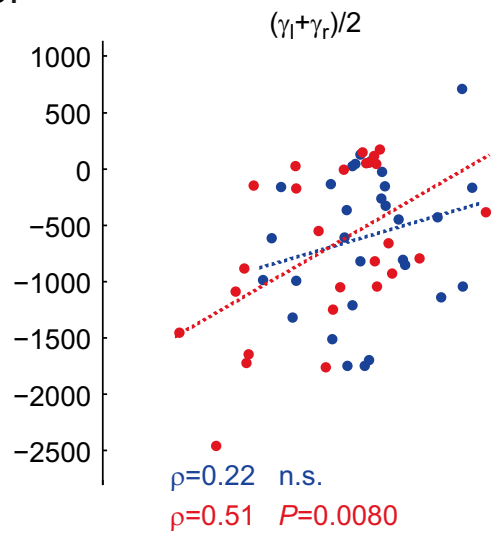

Cii

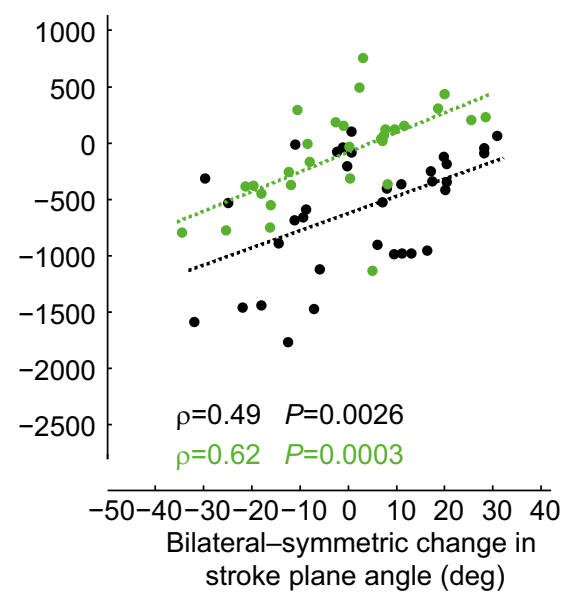

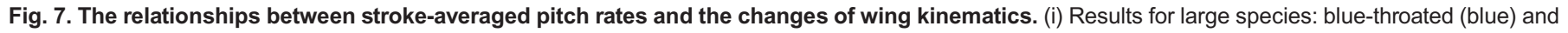
magnificent (red) hummingbirds. (ii) Results for small species: black-chinned (black) and broad-billed (green) hummingbirds. Stroke-averaged pitch rate as functions of $(\mathrm{A})$ bilateral-symmetric change in wing spanwise rotation $\left(\psi_{\mathrm{I}}+\psi_{\mathrm{r}}\right) / 2$, (B) bilateral-symmetric change in wing velocity $\left(U_{\mathrm{I}}+U_{\mathrm{r}}\right) / 2$ and $(\mathrm{C})$ bilateralsymmetric change in stroke plane angle $\left(\gamma_{1}+\gamma_{r}\right) / 2$. The subscripts I and $r$ denote left and right wings, respectively. Dotted lines represent the best-fit of the data through linear regression. The Pearson's linear correlation coefficient and $P$-value (double-tail Student's $t$ distribution) between each pair of pitch rates and wing kinematic changes are shown at the bottom of each graph.

hovering hummingbirds carrying weights (Chai et al., 1997) or with progressively reduced air density (Chai and Dudley, 1995). However, in display dives, male black-chinned hummingbirds almost doubled their wingbeat frequency (reached $93 \mathrm{~Hz}$ ) (Feo and Clark, 2010). In load lifting, hummingbirds rely primarily on increased stroke amplitude to augment lift (Chai et al., 1997; Chai and Dudley, 1995). A major difference between the load-lifting experiment and the present experiment was that the former usually lasted longer periods of time, as the birds were required to take off by overcoming gravity and then sustain hovering for approximately $1 \mathrm{~s}$, while the duration of escape manoeuvres in our study was $\sim 0.15 \mathrm{~s}$. At least within the few wingbeats for generating higher manoeuvring forces and moments, the hummingbirds in our study appeared capable of boosting muscle mass-specific power to a substantially higher level than previously observed (Cheng et al., 2016).

\section{Wing kinematics for roll rotation}

In the second half of escape manoeuvres, a rapid body roll was critical for reorientation of flight direction and recovery to stable forward or hovering flight. This was achieved by the bilateralasymmetric wing kinematic changes in wing deviation, spanwise rotation and velocity (roll pattern, Fig. 6, Table S3), all of which had significant correlations with the body roll rates (Fig. 8). A bilateral difference in wing deviation was conspicuous among these changes, especially for larger species (Fig. 6A,B). A bird elevated the outer wing and lowered the inner wing so that the normal axis of the stroke plane $\left(x_{\mathrm{s}}\right)$ was tilted laterally towards the inner wing, or in the direction of rolling. Similar to the stroke plane tilt in the pitch rotation, such a bilateral difference of wing motion likely created a lift-based roll moment and a lateral force directed from the outer wing to the inner wing. Note that lateral force may be used for weight support to prevent any significant altitude loss when the body is oriented horizontally with one side down (e.g. posture 6 in Fig. 2B). Similar bilateral-asymmetric changes in the wing deviation and stroke plane angle have been observed in significantly slower yaw turns in Anna's hummingbirds (Calypte anna) as they fed continuously from a revolving artificial feeder (Altshuler et al., 2012; Read et al., 2016).

Bilateral differences of wing spanwise rotation corresponded to reduced pronation and enhanced supination for the inner wing so that its stroke-averaged lift was reoriented backwards and was more perpendicular to the body longitudinal axis. Conversely, strokeaveraged lift of the outer wing was tilted forward and was more 

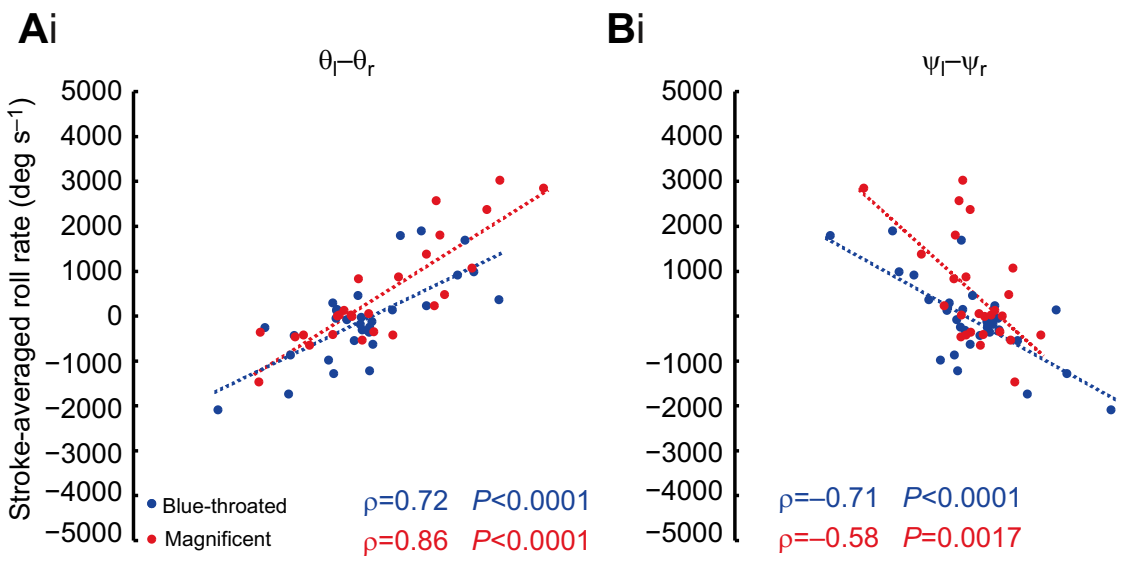

Fig. 8. The relationships between strokeaveraged roll rates and the changes of wing kinematics. (i) Results for large species: bluethroated (blue) and magnificent (red) hummingbirds. (ii) Results for small species: blackchinned (black) and broad-billed (green) hummingbirds. Stroke-averaged roll rate as functions of $(A)$ bilateral difference in wing deviation $\left(\theta_{\mathrm{l}}-\theta_{\mathrm{r}}\right)$ and $(\mathrm{B})$ bilateral difference in wing spanwise rotation $\left(\psi_{1}-\psi_{\mathrm{r}}\right)$. Other definitions are as in Fig. 7.

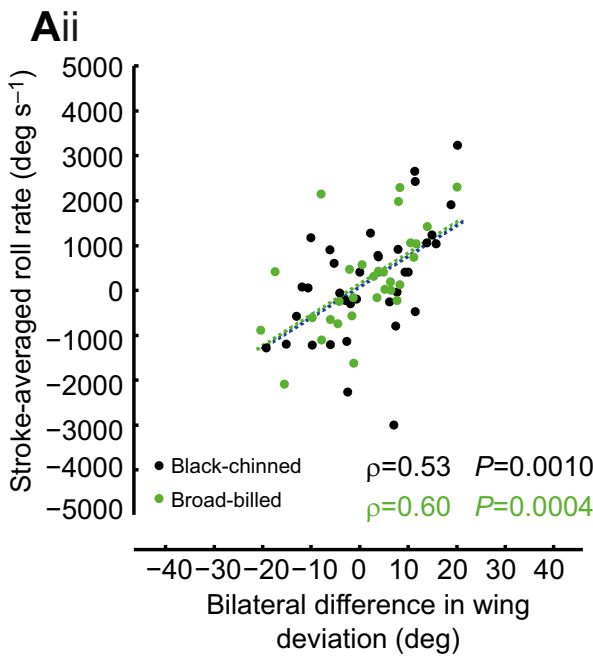

Bii

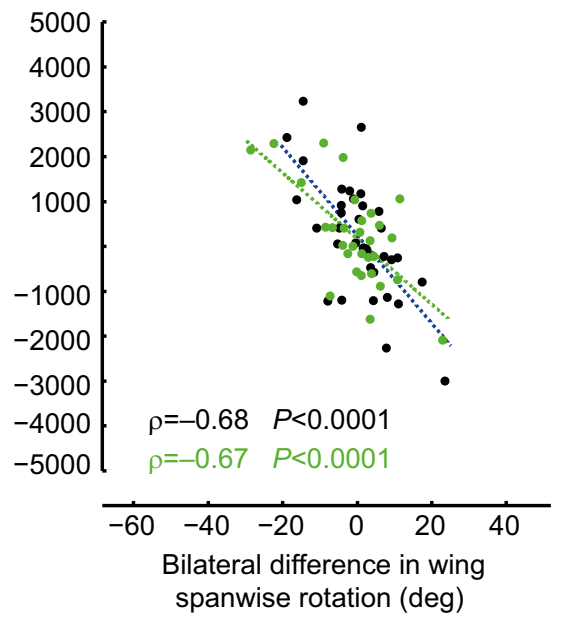

parallel to the body longitudinal axis. This asymmetry likely created a lift-based roll moment as well as a coupled yaw moment. Finally, the bilateral difference of wing stroke amplitude (Fig. 6) and, correspondingly, the wing velocity led to an imbalance of lift between the inner and outer wings, and therefore also created a liftbased roll moment. Bilateral-asymmetric changes in the wing stroke amplitude were also observed by Altshuler et al. (2012).

Roll moment was mostly created during the downstrokes, when total force was mostly perpendicular to the body longitudinal axis; during the upstroke, the total force was approximately parallel to the longitudinal body axis, and was thus less effective in creating roll moment, but more effective in creating yaw moment; this interpretation is confirmed through aerodynamic analysis (Cheng et al., 2016). Statistical analysis shows that for all four species, only downstroke-averaged wing kinematic variables have significant correlations with roll rates (Tables S1, S3).

\section{Wing kinematics for yaw rotation}

Escape manoeuvres were mainly composed of large pitch and roll rotations, and yaw rotation was less conspicuous. However, a minor (positive) yaw rotation during the initial pitching phase assisted the alignment of the body longitudinal axis to the direction of escape (Fig. 2D,H, 3D,J). For larger species (Figs 2D, 3D), we also found that, in addition to the dominant roll rotation, a (negative) yaw rotation helped to reorient the body back to a normal hovering or forward flight posture. Yaw moment was mainly created in the upstroke from bilateral differences in wing spanwise rotation, which were also concurrent with roll moment. There was significant correlation between the yaw rate and the bilateral difference of wing spanwise rotation averaged over the upstroke but not over the downstroke (Table S4).

Wing kinematic variables averaged over the downstrokes had more-significant correlations with the body kinematics than those averaged over the upstrokes (except for yaw), thus revealing the crucial role of downstroke in the control of escape manoeuvres (Warrick et al., 2005, 2009); this is further confirmed through aerodynamic analysis (Cheng et al., 2016). This result is also consistent with the twofold difference in muscle mass between pectoralis major and supracoracoideus in hummingbirds (Hartman, 1961).

\section{DISCUSSION}

\section{Comparing the manoeuvres of fruit flies and hummingbirds}

Despite differences in size and biological design, escape manoeuvres of hummingbirds in response to looming stimulus were similar to those of fruit flies in terms of peak roll and pitch rates (Muijres et al., 2014) and peak angular rates during voluntary yaw manoeuvres (Hedrick et al., 2009). The similar rotational manoeuvrability between these two taxa is a result of complex interactions of neural, physiological and dynamic processes that were independently shaped by evolutionary and ecological demands. However, wing manoeuvring kinematics of these two taxa show that the underlying dynamics, without considering the neural and physiological processes, are quite different. Hummingbirds used greater changes of wing kinematics than fruit flies; this was mainly due to the broad scaling effect that favours 
smaller fliers, allowing them to generate higher angular velocity and acceleration than their larger counterparts (Kumar and Michael, 2012). This is also apparent in power expenditures of larger and smaller hummingbirds (Cheng et al., 2016). For animals as small as fruit flies, if they were to use similar amounts of change in wing kinematics as hummingbirds, the accelerations generated would quickly destabilize their flight, and their sensorimotor system may not respond rapidly enough to ensure a stable flight (Chang and Wang, 2014). Fruit flies only use subtle wing kinematic changes in free-flight manoeuvres (Bergou et al., 2010; Fry et al., 2003; Muijres et al., 2015) except when being forced into extreme flight conditions with significantly higher body rates than those found in the free-flight manoeuvres (Beatus et al., 2015).

Although subtle wing kinematic changes are sufficient for generating rotational moments in fruit flies, this may limit their ability to change the directions of aerodynamic forces. Even during the fastest escape manoeuvres, the total aerodynamic force vector of a fruit fly changes very little relative to the body, such that a 'helicopter model' is a valid descriptor (Muijres et al., 2015). For hummingbirds, large changes in wing kinematic angles indicate that they modulate force vectors relative to their body, and, therefore, do not conform to the 'helicopter model'. This is confirmed via estimating the aerodynamic force vector based on the body acceleration and gravity, and then calculating its longitudinal (Fig. 3G,O) and lateral (Fig. 3H,P) tilt angles. From the plot, we can see that there is a large backward tilt of force vector in the pitching phase of the escape manoeuvre, at least $35 \mathrm{deg}$ for the magnificent hummingbird and $60 \mathrm{deg}$ for the black-chinned hummingbird. This is further confirmed via detailed aerodynamic force calculation (Cheng et al., 2016). As a result, hummingbirds may create manoeuvring forces independent of their body angles and enjoy enhanced linear manoeuvrability compared with smaller animals. This could be a significant locomotive advantage for them when preying on insects.

\section{Head saccades}

The start of body roll at the second half of manoeuvre was concurrent with a rapid head saccade towards the escape direction (Figs 2, 3). Head saccades lasted approximately $40-50 \mathrm{~ms}$ (Fig. 3F,N) with a magnitude close to $150 \mathrm{deg}$, and finished prior to the completion of body roll rotation (also see Fig. 2B,F). This corresponds to an average saccade rate higher than $3000 \mathrm{deg} \mathrm{s}^{-1}$, which is slightly faster than the peak head-saccade rate reported in lovebirds (Agapornis roseicollis) and significantly faster than those reported for other vertebrates (Kress et al., 2015). In the entire manoeuvre, except the rapid-saccade phase, the head maintained a highly stable orientation with respect to the global frame in spite of the body rotation, putatively owing to the bird's vestibular system (Haque and Dickman, 2005; Money and Correia, 1972). As a result, the stability of head orientation and the short duration of head saccade may contribute to attaining a stable gaze and reducing the period of visual blur, thereby enhancing visual feedback for high-level planning of the subsequent flight, for example, to stop and avoid running into the wall of the flight chamber. In contrast, because the head saccade preceded completion of body roll, the roll control at the second half of the manoeuvre was unlikely to be regulated directly by the visual feedback. Assuming stabilized head/vision, one hypothesis that merits further study is that the proprioception from the neck (Necker, 2001) was used to regulate the body roll angle towards the end of the manoeuvre (by afferent encoding of muscle strain between the head and the body). Although putative pathways are known (Necker, 2001), the function of such pathways in flight has not been studied. An analogous pattern for proprioception for flight control is used in various insects with cuticular hairs located at their prothorax that detect head angle, including dragonflies (Mittelstaedt, 1950), bluebottle flies (Liske, 1977) and locusts (Taylor, 1981).

\section{Acknowledgements}

We thank the director and staff at the Southwestern Research Station (under the direction of the American Museum of Natural History, New York, USA) for hosting our research efforts; Joana Figuière, Kathleen Langland, Rebecca Schroeder and Joseph Canepa for assisting with bird capture; Yi Wang and Stephen Van Kooten for assisting in extraction of wing kinematics and drawing some schematic figures; and two anonymous reviewers for providing insightful suggestions that helped us improve the quality of the manuscript.

Competing interests

The authors declare no competing or financial interests.

\section{Author contributions}

B.C., B.W.T., G.T.C.C. and X.D. contributed to the design of experiments, B.C. B.W.T., D.R.P. and S.M.W. prepared the experiments, and B.C. and B.W.T. performed the experiments. All authors contributed to the interpretation of findings and preparation of the manuscript, and B.C., B.W.T. and T.L.H. contributed to the data analysis.

\section{Funding}

Funding was provided by the National Science Foundation [NSF CMMI 1234737 to X.D., B.W.T. and T.L.H.] and the National Aeronautics and Space Administration [Climate and Biological Response 10-BIOCLIM10-0094 to D.R.P.].

\section{Supplementary information}

Supplementary information available online at

http://jeb.biologists.org/lookup/doi/10.1242/jeb.137539.supplemental

\section{References}

Altshuler, D. L., Dudley, R. and McGuire, J. A. (2004). Resolution of a paradox: hummingbird flight at high elevation does not come without a cost. Proc. Natl. Acad. Sci. USA 101, 17731-17736.

Altshuler, D., Dudley, R., Heredia, S. and McGuire, J. (2010). Allometry of hummingbird lifting performance. J. Exp. Biol. 213, 725-734.

Altshuler, D. L., Quicazán-Rubio, E. M., Segre, P. S. and Middleton, K. M. (2012) Wingbeat kinematics and motor control of yaw turns in Anna's hummingbirds (Calypte anna). J. Exp. Biol. 215, 4070-4084.

Anderson, J. (2005). Fundamentals of Aerodynamics. New York, NY: McGraw-Hil Higher Educationl.

Beatus, T., Guckenheimer, J. M. and Cohen, I. (2015). Controlling roll perturbations in fruit flies. J. R. Soc. Interface 12, 20150075.

Bergou, A. J., Ristroph, L., Guckenheimer, J., Cohen, I. and Wang, Z. J. (2010) Fruit flies modulate passive wing pitching to generate in-flight turns. Phys. Rev. Lett. 104, 148101.

Chai, P. and Dudley, R. (1995). Limits to vertebrate locomotor energetics suggested by hummingbirds hovering in heliox. Nature 377, 722-725.

Chai, P. and Dudley, R. (1996). Limits to flight energetics of hummingbirds hovering in hypodense and hypoxic gas mixtures. J. Exp. Biol. 199, 2285-2295.

Chai, P. and Millard, D. (1997). Flight and size constraints: hovering performance of large hummingbirds under maximal loading. J. Exp. Biol. 200, 2757-2763.

Chai, P., Chen, J. and Dudley, R. (1997). Transient hovering performance of hummingbirds under conditions of maximal loading. J. Exp. Biol. 200, 921-929.

Chang, S. and Wang, Z. J. (2014). Predicting fruit fly's sensing rate with insect flight simulations. Proc. Natl. Acad. Sci. USA 111, 11246-11251.

Cheng, B., Deng, X. and Hedrick, T. L. (2011). The mechanics and control of pitching manoeuvres in a freely flying hawkmoth (Manduca sexta). J. Exp. Biol. 214, 4092-4106.

Cheng, B., Tobalske, B. W., Powers, D. R., Hedrick, T. L., Wang, Y., Wethington S. M., Chiu, G. T.-C. and Deng, X. (2016). Flight mechanics and control of escape manoeuvres in hummingbirds. II. Aerodynamic force production, flight control and performance limitations. J. Exp. Biol. 219, 3532-3543.

Clark, C. J. (2009). Courtship dives of Anna's hummingbird offer insights into fligh performance limits. Proc. R. Soc. B Biol. Sci. 276, 3047-3052.

Clark, C. J. (2010). The evolution of tail shape in hummingbirds. Auk 127, 44-56. Clark, C. J. (2011). Effects of tail length on an escape maneuver of the red-billed streamertail. J. Ornithol. 152, 397-408.

Dudley, R. (2000). The Biomechanics of Insect Flight. Princeton, NJ: Princeton University Press. 
Ellington, C. P. (1984). The aerodynamics of hovering insect flight. III. Kinematics. Philos. Trans. R. Soc. Lond. B. Biol. Sci. 305, 41-78.

Ellington, C. (1985). Power and efficiency of insect flight muscle. J. Exp. Biol. 115 293-304

Elzinga, M. J., Dickson, W. B. and Dickinson, M. H. (2012). The influence of sensory delay on the yaw dynamics of a flapping insect. J. R. Soc. Interface $\mathbf{9}$, 1685-1696.

Feo, T. J. and Clark, C. J. (2010). The displays and sonations of the black-chinned hummingbird (Trochilidae: Archilochus alexandri). Auk 127, 787-796.

Flanagan, J. R. and Wing, A. M. (1997). The role of internal models in motion planning and control: evidence from grip force adjustments during movements of hand-held loads. J. Neurosci. 17, 1519-1528.

Fry, S. N., Sayaman, R. and Dickinson, M. H. (2003). The aerodynamics of freeflight maneuvers in Drosophila. Science 300, 495-498.

Fuller, S. B., Straw, A. D., Peek, M. Y., Murray, R. M. and Dickinson, M. H. (2014) Flying Drosophila stabilize their vision-based velocity controller by sensing wind with their antennae. Proc. Natl. Acad. Sci. USA 111, E1182-E1191.

Greenewalt, C. H. (1962). Dimensional relationships for flying animals. Smithsonian Misc. Collect. 144, 1-46.

Haque, A. and Dickman, J. D. (2005). Vestibular gaze stabilization: different behavioral strategies for arboreal and terrestrial avians. J. Neurophysiol. 93 1165-1173.

Hartman, F. A. (1961). Locomotor Mechanisms of Birds. Washington, DC: Smithsonian Institution.

Hedrick, T. L. (2008). Software techniques for two- and three-dimensional kinematic measurements of biological and biomimetic systems. Bioinspir. Biomim. 3 034001.

Hedrick, T. L., Cheng, B. and Deng, X. (2009). Wingbeat time and the scaling of passive rotational damping in flapping flight. Science 324, 252-255.

Hedrick, T. L., Tobalske, B. W., Ros, I. G., Warrick, D. R. and Biewener, A. A (2011). Morphological and kinematic basis of the hummingbird flight stroke: scaling of flight muscle transmission ratio. Proc. R. Soc. B Biol. Sci 279, 1986-1992.

Iwaniuk, A. N. and Wylie, D. R. W. (2007). Neural specialization for hovering in hummingbirds: hypertrophy of the pretectal nucleus lentiformis mesencephali. J. Comp. Neurol. 500, 211-221.

Jackson, B. E. and Dial, K. P. (2011). Scaling of mechanical power output during burst escape flight in the Corvidae. J. Exp. Biol. 214, 452-461.

Josephson, R. K., Malamud, J. G. and Stokes, D. R. (2000). Asynchronous muscle: a primer. J. Exp. Biol. 203, 2713-2722.

Keennon, M., Klingebiel, K., Won, H. and Andriukov, A. (2012). Development of the nano hummingbird: a tailless flapping wing micro air vehicle. In 50th AIAA Aerospace Sciences Meeting Including the New Horizons Forum and Aerospace Exposition, Nashville, TN, pp. 9-12

Kress, D., van Bokhorst, E. and Lentink, D. (2015). How lovebirds maneuver rapidly using super-fast head saccades and image feature stabilization. PLoS ONE 10, e0129287.

Kumar, V. and Michael, N. (2012). Opportunities and challenges with autonomous micro aerial vehicles. Int. J. Robotics Res. 31, 1279-1291.

Leishman, J. G. (2006). Principles of Helicopter Aerodynamics. New York: Cambridge University Press.

Liske, E. (1977). The influence of head position on the flight behaviour of the fly, Calliphora erythrocephala. J. Insect Physiol. 23, 375-379.

Liu, Y. and Sun, M. (2008). Wing kinematics measurement and aerodynamics of hovering droneflies. J. Exp. Biol. 211, 2014-2025.

Ma, K. Y., Chirarattananon, P., Fuller, S. B. and Wood, R. J. (2013). Controlled flight of a biologically inspired, insect-scale robot. Science 340, 603-607.

Marden, J. H. (1994). From damselflies to pterosaurs: how burst and sustainable flight performance scale with size. Am. J. Physiol. 266, R1077-R1077.

Mischiati, M., Lin, H.-T., Herold, P., Imler, E., Olberg, R. and Leonardo, A (2015). Internal models direct dragonfly interception steering. Nature $\mathbf{5 1 7}$ 333-338
Mittelstaedt, H. (1950). Physiologie des Gleichgewichtssinnes bei fliegenden Libellen. J. Comp. Physiol. A Neuroethol. Sens. Neural Behav. Physiol. 32 , 422-463.

Money, K. E. and Correia, M. J. (1972). The vestibular system of the owl. Comp. Biochem. Physiol. A Physiol. 42, 353-358.

Muijres, F. T., Elzinga, M. J., Melis, J. M. and Dickinson, M. H. (2014). Flies evade looming targets by executing rapid visually directed banked turns. Science 344 , 172-177.

Muijres, F. T., Elzinga, M. J., Iwasaki, N. A. and Dickinson, M. H. (2015). Body saccades of Drosophila consist of stereotyped banked turns. J. Exp. Biol. 218 864-875.

Murray, R. M., Li, Z. and Sastry, S. S. (1994). A Mathematical Introduction to Robotic Manipulation. Boca Raton, FL: CRC Press.

Necker, R. (2001). Spinocerebellar projections in the pigeon with special reference to the neck region of the body. J. Comp. Neurol. 429, 403-418.

Read, T. J. G., Segre, P. S., Middleton, K. M. and Altshuler, D. L. (2016) Hummingbirds control turning velocity using body orientation and turning radius using asymmetrical wingbeat kinematics. J. R. Soc. Interface 13, 20160110.

Ristroph, L., Bergou, A. J., Ristroph, G., Coumes, K., Berman, G. J. Guckenheimer, J., Wang, Z. J. and Cohen, I. (2010). Discovering the flight autostabilizer of fruit flies by inducing aerial stumbles. Proc. Natl. Acad. Sci. USA 107, 4820-4824

Ristroph, L., Ristroph, G., Morozova, S., Bergou, A. J., Chang, S., Guckenheimer, J., Wang, Z. J. and Cohen, I. (2013). Active and passive stabilization of body pitch in insect flight. J. R. Soc. Interface 10, 20130237.

Roll, J. A., Cheng, B. and Deng, X. (2015). An electromagnetic actuator for highfrequency flapping-wing micro air vehicles. IEEE Trans. Robot. 31, 400-414.

Russell, S. M. and Russell, R. O. (2001). The North American Banders'Manual for Banding Hummingbirds. Point Reyes Station, CA: North American Banding Council

Scanes, C. G. (2014). Sturkie's Avian Physiology. New York: Elsevier.

Segre, P. S., Dakin, R., Zordan, V. B., Dickinson, M. H., Straw, A. D. and Altshuler, D. L. (2015). Burst muscle performance predicts the speed acceleration, and turning performance of Anna's hummingbirds. eLife 4, e11159.

Song, J., Luo, H. and Hedrick, T. L. (2014). Three-dimensional flow and lift characteristics of a hovering ruby-throated hummingbird. J. R. Soc. Interface 11 20140541

Sprayberry, J. D. H. (2009). Responses of descending visually-sensitive neurons in the hawkmoth, Manduca sexta, to three-dimensional flower-like stimuli. J. Insect Sci. 9,7 .

Taylor, C. P. (1981). Contribution of compound eyes and ocelli to steering of locusts in flight: II. Timing changes in flight motor units. J. Exp. Biol. 93, 19-31.

Taylor, G. K. (2001). Mechanics and aerodynamics of insect flight control. Biol. Rev. 76, 449-471.

Tobalske, B. W., Peacock, W. L. and Dial, K. P. (1999). Kinematics of flapbounding flight in the zebra finch over a wide range of speeds. J. Exp. Biol. 202 1725-1739.

Tobalske, B. W., Hedrick, T. L., Dial, K. P. and Biewener, A. A. (2003) Comparative power curves in bird flight. Nature 421, 363-366.

Tobalske, B. W., Biewener, A. A., Warrick, D. R., Hedrick, T. L. and Powers, D. R. (2010). Effects of flight speed upon muscle activity in hummingbirds. J. Exp. Biol. 213, 2515-2523.

Walker, S. M., Thomas, A. L. R. and Taylor, G. K. (2009). Deformable wing kinematics in free-flying hoverflies. J. R. Soc. Interface 7, 131-142.

Warrick, D. R., Tobalske, B. W. and Powers, D. R. (2005). Aerodynamics of the hovering hummingbird. Nature 435, 1094-1097.

Warrick, D. R., Tobalske, B. W. and Powers, D. R. (2009). Lift production in the hovering hummingbird. Proc. R. Soc. B Biol. Sci. 276, 3747-3752.

Welch, K. C., Jr and Altshuler, D. L. (2009). Fiber type homogeneity of the flight musculature in small birds. Comp. Biochem. Physiol. B Biochem. Mol. Biol. 152 324-331.

Wilkerson, R. C. and Butler, J. F. (1984). The Immelmann turn, a pursuit maneuver used by hovering male Hybomitra hinei wrighti (Diptera: Tabanidae). Ann. Entomol. Soc. Am. 77, 293-295. 